\title{
Gays Don't Cause Storms
}

\author{
Aidan Bissell-Siders
}

April 1, 2018

\section{Abstract}

The hypothesis that same-sex couples cause storm damages is examined and rejected.

\section{Introduction}

An increasing number of storms slam into the helpless United States. Why? In the aftermath of Hurricane Harvey, conservative Christian intellectuals sought to answer.

Wiles (2017) upheld a causal link: "Here's a city that has boasted of its LGBT devotion, its affinity for the sexual perversion movement in America. They're underwater." [1] Swanson (2017) agreed: "Jesus sends the message home: unless Americans repent, unless Houston repents, unless New Orleans repents, they will all likewise perish." 2] In dissent, Coulter (2017) took a balanced view: "I don't believe Hurricane Harvey is GoD's punishment for Houston electing a lesbian mayor. But that is more credible than 'climate change'." 3

For some authors, it is "unscientific" to blame gays for storm events. 4] However, we contend that Colbert (2012) provides a falsifiable framework: "Hurricanes form from rising moisture created by hot, steamy man-action aboard a gay Caribbean cruise. When that sin gets high enough, it makes the angels cry, and those tears fall to the earth in the form of massive precipitation." 5

\section{Literature Review}

This "gay-storm hypothesis" has obvious significance for civil defense: Tropical cyclones alone cost the US an estimated $\$ 400$ billion between 1980 and 2014. [6] Thus, we conducted a thorough review of the literature, produced below:

\begin{tabular}{l|l}
\hline Reference & Results \\
\hline Walworth & $\begin{array}{l}\text { 1: Negative correlation between gay political } \\
\text { groups per capita and tornadoes } \\
\text { 2: Positive correlation between number of } \\
\text { Protestants and tornadoes }\end{array}$ \\
\hline Burnett & $\begin{array}{l}\text { More gay weddings means more weddings, } \\
\text { which means more tears of joy, which means } \\
2014[8]\end{array}$ \\
\hline Warren \& & $\begin{array}{l}\text { Significantly more hurricanes occur in states } \\
\text { Without legal same-sex marriage (and states }\end{array}$ \\
$2016[9]$ & that vote for Republican presidents) \\
\hline
\end{tabular}

Figure 1: Summary of the gay-storm literature

The scientific literature is evidently rife with debate between gay-storm hypothesis supporters and critics.

\section{Hypotheses}

We hope to provide clarity to this stormy ${ }^{1}$ debate. We extend Colbert's analysis (more gayness $=$ more storms) and posit two testable hypotheses:

\section{H1: Higher gayness causes higher storm damages.}

H2: Higher godliness causes lower storm damages.

\section{Data Sources}

Gayness and Godliness: Census Bureau: Unfortunately, we cannot measure gayness directly! 2 Fortunately, we do know the mere existence of same-sex couples "will quickly destroy the traditional famil[ies]" 10 GOD is quite a fan of.

As a proxy for gayness (and the innocent, hard-working marriages it has destroyed), we produce a weighted proportion of same-sex couples (SSC) to total couples ${ }^{3}$ If gayness incurs GOD's wrath, then HE would presumably punish gayness harder (H1) than His tireless servants (H2). To test this, we produce a weighted proportion of Religious Service Employment (RSE) relative to total employment!

We estimate both measures with 2000-16 data from the American Community Survey (ACS), a nationallyrepresentative household survey.

Storm events: National Climatic Data Center: The Storm Events Database (SED) estimates damages for natural hazards that caused casualties, property damage, or commercial disruption. 11] Though "small" hazard estimates have noted problems, 12, 13] we have no reason to doubt the broad accuracy of SED estimates. Given that an angrier GOD would presumably send stronger storms, we use storm damages per capita as a proxy for His wrath.

Population: Census Bureau: The Census provides intercensal data for 2000-09 14 and population estimates for 2010-16. 15] Blessed be the fruit!

\section{Analysis}

We used R 3.4.1[16] with RStudio 1.0.153[17] and many packages. 18] Following Open Science Collaboration reproducibility standards, 19] we publicize data, code \& figures at osf.io/yujdz. Code is also included in Appendix A

We calculate total storm damage (sed_dt.rds), population (pop_dt.rds), and weighted proportions for SSC and RSE (acs_summary_dt.rds) for each state and DC, 2000-16. We produce scatterplots of storm damage/capita for all years in Figs. 2-3 and for state averages in 4-5; a US chloropleth map of state SSC and RSE averages in Fig. 7; and lineplots for each variable in Fig. 9. 


\section{Results}

Linear regression: We test our hypotheses via mixedmodel linear regression. Our left-hand variable is the decimal $\log \left(\log _{10}\right)$ of damages/capita. Our right-hand fixed effects are same-sex couples (SSC) or religious service employment (RSE). Our right-hand random effects are year and state. This model is expressed below for SSC:20]

$\log _{10}($ damages $/$ capita $)=\mathrm{SSC}+(1+\mathrm{SSC} \mid \mathrm{STATE})+(1+\mathrm{SSC} \mid \mathrm{YEAR})$

We plot these regressions in Figs. 2-3 and present our summary statistics below:

\begin{tabular}{rrr|rrrrr}
\hline & slope & int & AIC & BIC & $\chi^{2}$ & $\mathrm{p}$ & \\
\hline SSC & -0.27 & 1.17 & 2211 & 2254 & 3.1 & 0.07910 & \\
RSE & 1.05 & 0.19 & 2201 & 2243 & 18.2 & 0.00002 & $* * *$ \\
\hline
\end{tabular}

Figure 6: Linear mixed-effects model results

The correlation between same-sex couples and storm damages is not significant $(p=0.079)$. In contrast, the correlation between religious service employment and storm damages $(p=0.0002)$ is highly significant. 22 Both slopes, however, run contrary to the expectations of $H 1$ and H2. Our results

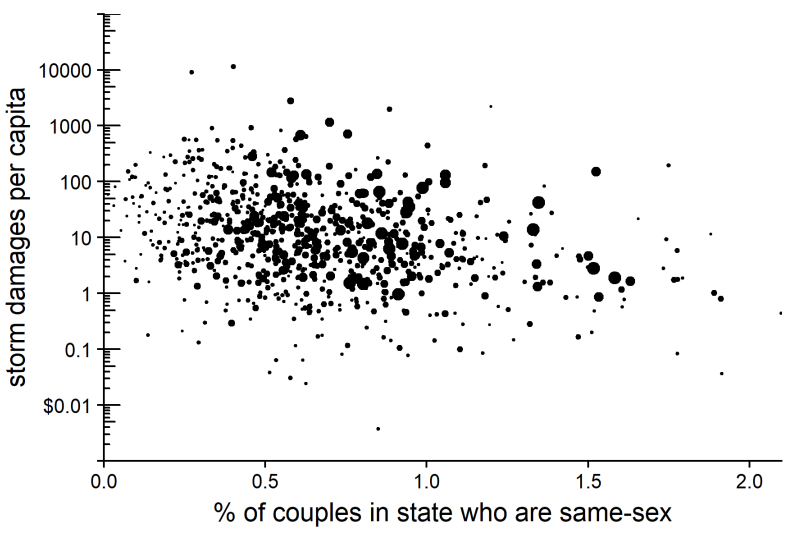

Figure 2: Same-sex couples vs storm damage in each US state (DC not shown), 2000-16. Size represents population.

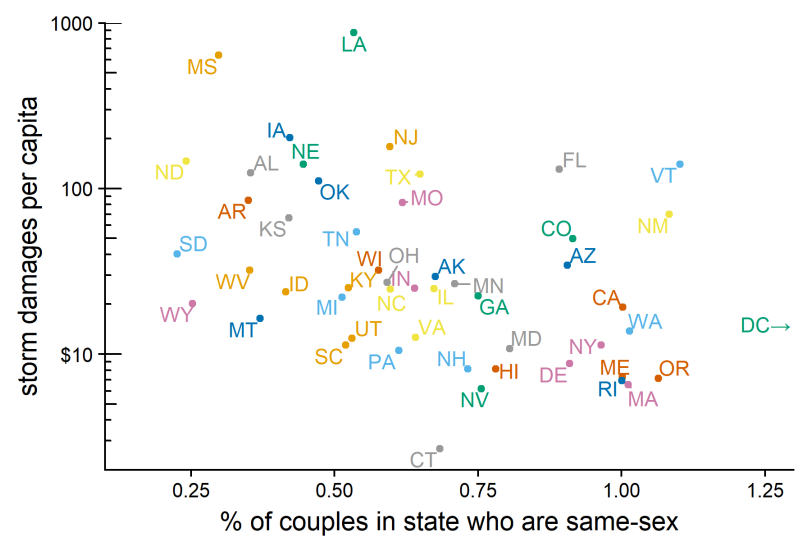

Figure 4: Average same-sex couples vs storm damage in each US state and DC, 2000-16. Not depicted: DC was located at 5.03 SSC and 17.4 damage/capita. suggest a $1 \%$ increase of same-sex couples would lead to a $56.4 \%$ decrease of storm damages/capita, while a $1 \%$ increase of religious service employment would lead to a $1186 \% \mathrm{in}$ crease of storm damages/capita! 6

Rank correlation: We examined states ranked by storm damages and SSC or RSE with Kendall's $\tau$ ? 7 Fig. 8 shows that, in all years, states with more same-sex couples had less storm damages (rejecting $H 1$ ) and states with more religious service employment had more storm damages (rejecting H2).

\section{Conclusions}

We confidently report that same-sex couples reduce storm damages while religious servicepersons increase them. It appears that, in His wisdom, GoD has let same-sex couples off the hook and instead directed His stormy 8 anger towards religious servicepersons for their failure to stop the gay scourge. (We also discuss a less-orthodox hypothesis in Appendix B.)

These results have clear policy implications: Civil defense planners should enforce state atheism and mandate gay marriages for all. 21] As a stopgap measure, states can create mobile teams of religious servicepersons to tempt divine storms towards low-population regions, where they can be safely dispersed by same-sex rapid responders.

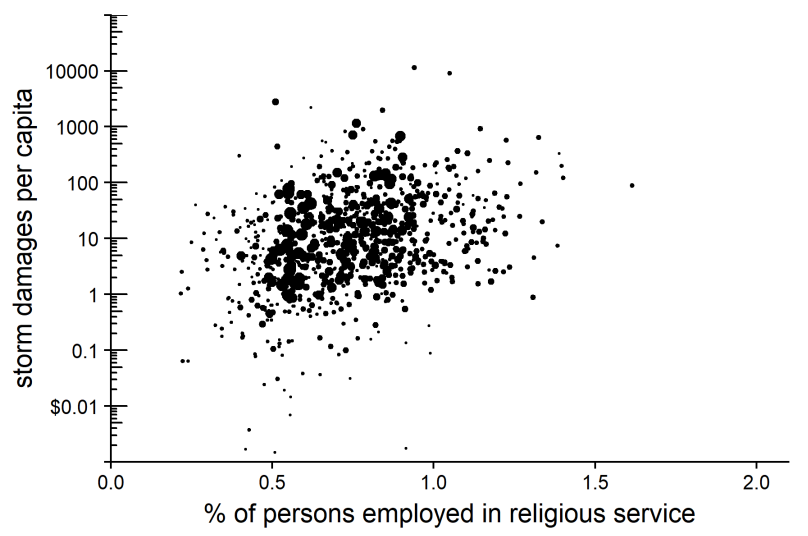

Figure 3: Religious service employment vs storm damage in each US state and DC, 2000-16. Size represents population.

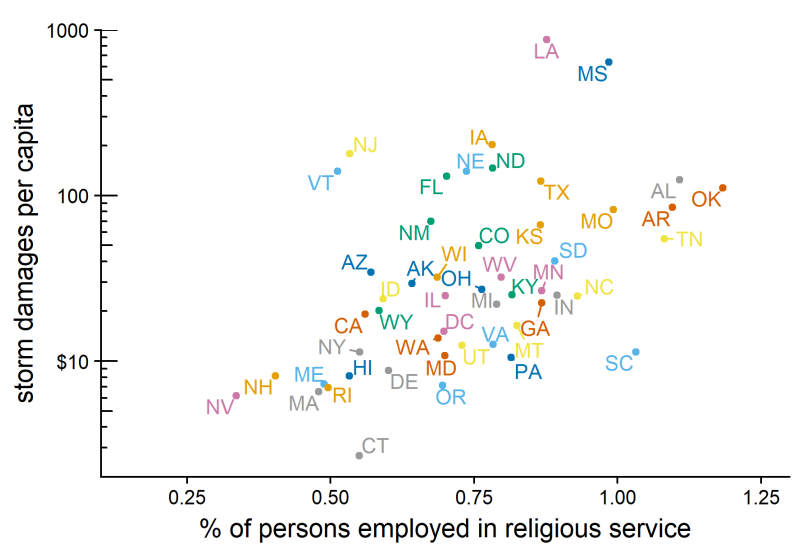

Figure 5: Average religious service employment vs storm damage in each US state and DC, 2000-16. 


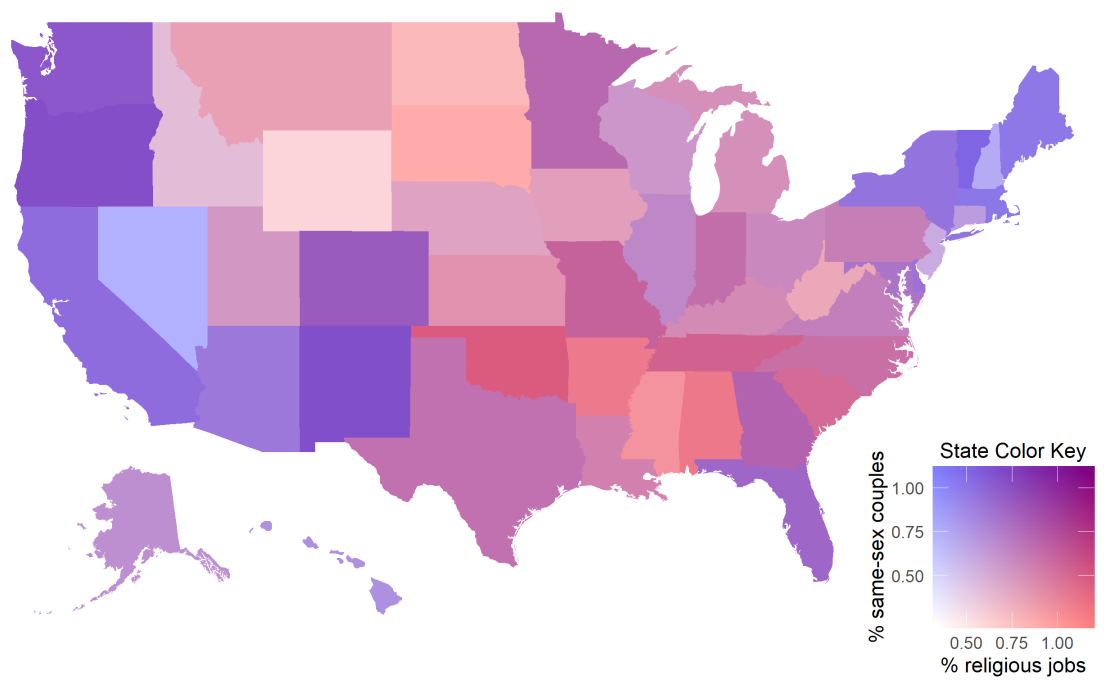

Figure 8: Chloropleth of state averages of both same-sex couples and religious service employment, 2000-16

We hope that your reaction to the above is "bullshit." We're not entirely sure why more same-sex couples live in low-storm-damage states and more religious servicepersons live in high-damage states. However, we know that states with low SSC and high RSE (see Fig. 8) tend to be Midwestern or Southeastern - the areas hardest hit by high-damage hurricanes 6 and tornadoes 23 . This suggests that the different average damage that different states have could better explain our results.

Indeed, when we add "state" as a fixed effect, same-sex couples reaches utter statistical insignificance $(\mathrm{p} \approx 1)$ while religious service employment becomes three orders of magnitude less significant than before $(\mathrm{p} \approx 0.02 \mathrm{vs} \mathrm{p} \approx 0.00002)$.

\begin{tabular}{rrr|rrrrr}
\hline & slope & int & AIC & BIC & $\chi^{2}$ & $\mathrm{p}$ & \\
\hline SSC & 0.00 & 1.21 & 2158 & 2444 & 0.0 & 0.99878 & \\
RSE & 0.67 & 0.78 & 2155 & 2441 & 5.1 & 0.02331 & $*$ \\
\hline
\end{tabular}

Figure 7: Linear mixed-effects model results

It's almost as if storms are natural events determined by climate and geography rather than GoD's wrath on Earth.

\section{Acknowledgements}

The authors declare no financial conflicts of interest (and is unsure how any could exist) and thanks Charles Delwiche, Gary Gates, and Joseph Heavner for their critical readings of the manuscript.

\section{Notes}

1 Please visit http://instantrimshot.com/

${ }_{2}$ We are also unaware of objective methods to evaluate a person's level of "sexual perversion" or ability to "destroy traditional families" - though we have high hopes for the future.

3 A SSC occurs when a householder "identifies another individual in the household aged 16 or older as 'husband/wife' or 'unmarried partner"' and both are the same sex.25 (Cohen 2011 suggests the terms "heterogamy" and "homogamy".[26]) Because opposite-sex couples (OSCs)

\begin{tabular}{l|rrl|rrl}
\hline & \multicolumn{3}{|c}{ \% same sex } & \multicolumn{3}{c}{ \% religious service } \\
year & $\tau$ & $\mathrm{p}$ & & $\tau$ & $\mathrm{p}$ & \\
\hline 2000 & -0.15 & 0.13294 & & 0.28 & 0.00414 & $* *$ \\
2001 & -0.20 & 0.03401 & $*$ & 0.38 & 0.00010 & $* * *$ \\
2002 & -0.34 & 0.00049 & $* * *$ & 0.27 & 0.00590 & $* *$ \\
2003 & -0.25 & 0.00957 & $* *$ & 0.30 & 0.00177 & $* *$ \\
2004 & -0.32 & 0.00095 & $* * *$ & 0.15 & 0.10958 & \\
2005 & -0.19 & 0.05423 & & 0.13 & 0.18553 & \\
2006 & -0.15 & 0.12476 & & 0.19 & 0.05423 & \\
2007 & -0.24 & 0.01324 & $*$ & 0.27 & 0.00561 & $* *$ \\
2008 & -0.13 & 0.18019 & & 0.21 & 0.02890 & $*$ \\
2009 & -0.32 & 0.00089 & $* * *$ & 0.38 & 0.00010 & $* * *$ \\
2010 & -0.17 & 0.08362 & & 0.18 & 0.06763 & \\
2011 & -0.16 & 0.09919 & & 0.20 & 0.04148 & $*$ \\
2012 & -0.22 & 0.02153 & $*$ & 0.14 & 0.13718 & \\
2013 & -0.28 & 0.00374 & $* *$ & 0.20 & 0.03834 & $*$ \\
2014 & -0.22 & 0.02153 & $*$ & 0.23 & 0.01657 & $*$ \\
2015 & -0.33 & 0.00075 & $* * *$ & 0.31 & 0.00141 & $* *$ \\
2016 & -0.35 & 0.00027 & $* * *$ & 0.29 & 0.00245 & $* *$ \\
\hline
\end{tabular}

Figure 9: Kendall's rank correlation results

far outnumber SSCs, even rare misreports of sex or relationship status among OSCs can substantially affect the count and demographics of SSCs. (Lofquist and Lewis 2015 linked Census and Social Security data to find that just $42.3 \%$ of married SS, $92.6 \%$ of unmarried SS, $97.5 \%$ of married OS, and $96.3 \%$ of unmarried OS couples were accurately reported. 27]) We followed Gates 2015[25]'s use of four filtering methods to remove false cases. Though Gates uses variables introduced in 2008, this does not substantially affect results (see Appendix C)

4. RSE is defined as INDP code 916 or 9160

5 Please visit http://lmgtfy.com/?q=blessed+be+the+fruit

6 The geometric mean of damage/capita is 0.9441. The slope of SSC is -0.2736 and RSE is 1.04924. A 1-unit increase of SSC becomes a $10^{1 \times 0.2736} / 0.9441=0.5641 \approx 56.2 \%$ decrease of storm damages per capita, and a 1-unit increase of RSE becomes a $10^{1 \times 1.04924} / 0.9441=$ $11.86 \approx 1186 \%$ increase of storm damages per capita.

7 Croux and Dehon 2010 suggest that Kendall's $\tau$ is virtually always superior to Spearman's $\rho$. 28

8 Please visit http://instantrimshot.com/ again. 
$\begin{array}{lllllllllllllll}\text { AL } & \text { AK } & \text { AZ } & \text { AR } & \text { CA } & \text { CO } & \text { CT } & \text { DE } & \text { FL } & \text { GA } & \text { HI } & \text { ID } & \text { IL }\end{array}$

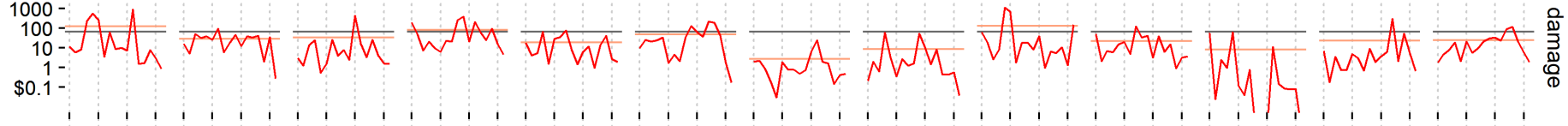

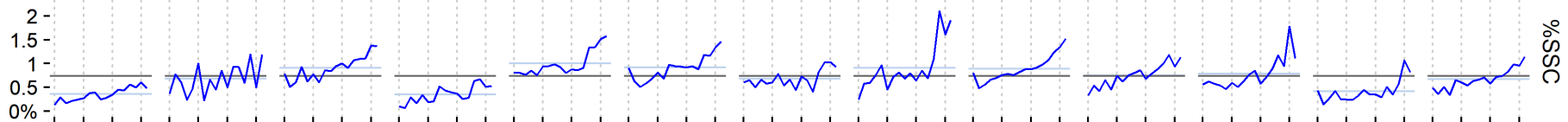
2 -

$1.5-$

$0.5-$

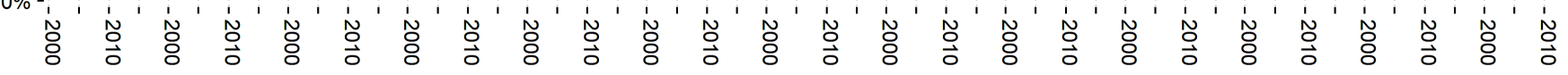
$\begin{array}{llllllllllllll}\text { IN } & \text { IA } & \text { KS } & \text { KY } & \text { LA } & \text { ME } & \text { MD } & \text { MA } & \text { MI } & \text { MN } & \text { MS } & \text { MO } & \text { MT }\end{array}$

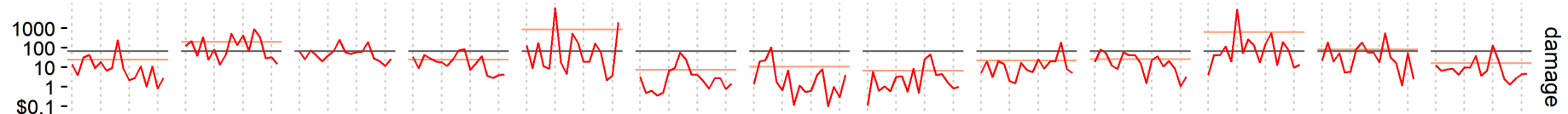

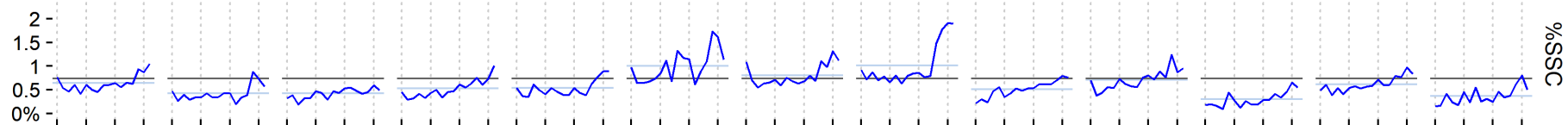
2 -

1.5 -

0.5

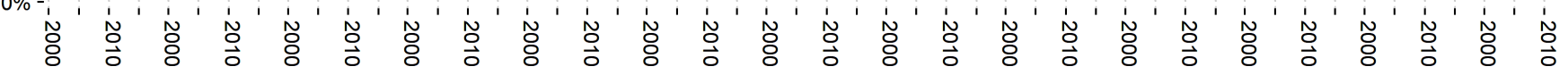

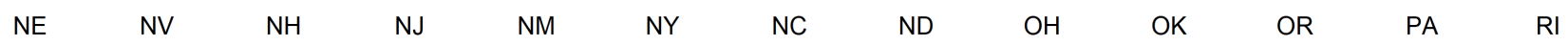

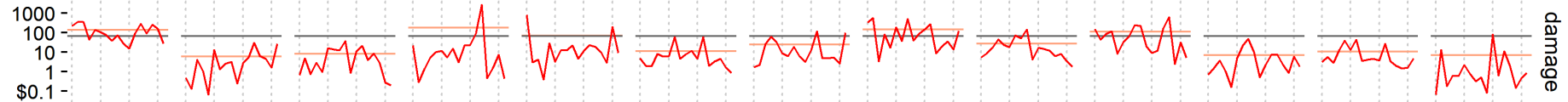

$2-$

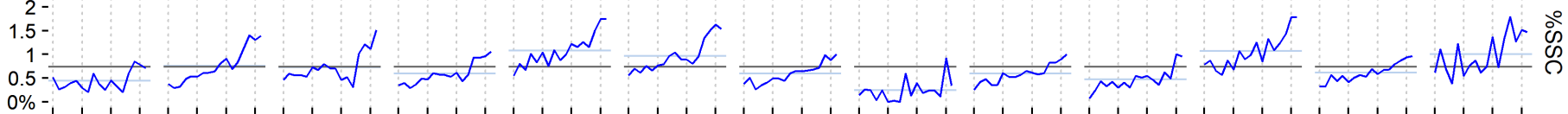
$2-$

$2-5$

$1-$

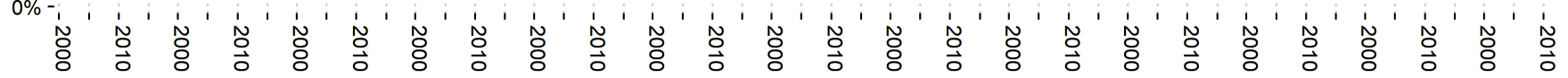
$\begin{array}{llllllllllllll}\text { SC } & \text { SD } & T N & T X & \text { UT } & \text { VT } & \text { VA } & \text { WA } & \text { WV } & \text { WI } & \text { WY } & \text { DC }\end{array}$

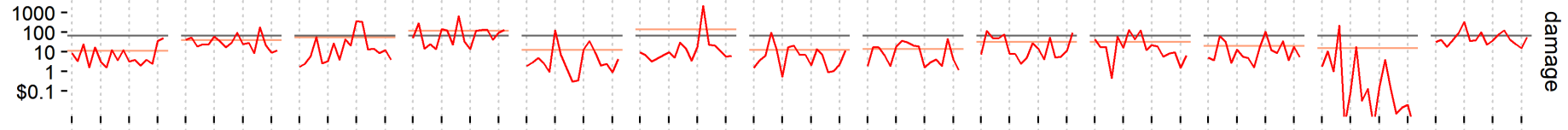

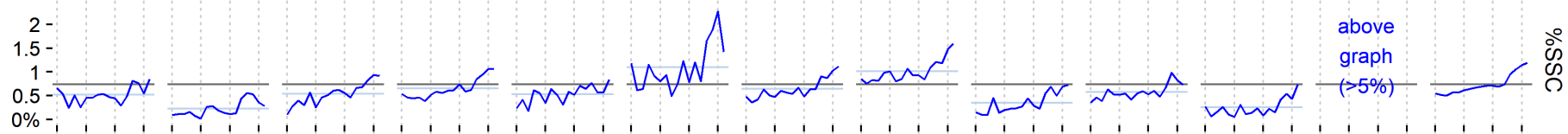

$2-$

$1.5-$

$1-5$

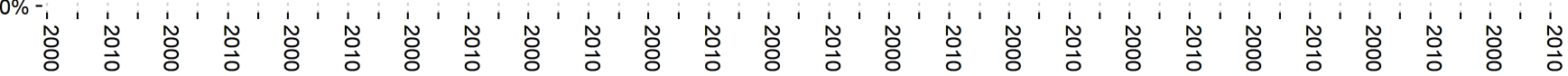

Figure 10: Same-sex couples, religious service, and damage/capita bin each US state and DC, 2000-2016. Light-colored lines are averages. (We use the triple facet style in hope of avoiding noted problems with multiscale line graphs [24].) 


\section{References}

[1] Wiles R. Houston Flooding: Climate Change or Morality Change? TRUNEWS. http://www.trunews.com/listen/houstonflooding-climate-change-or-morality-change Published 29 August 2017. Accessed 30 September 2017. See also: http://www.rightwingwatch.org/post/rick-wiles-hurricaneharvey-is-punishment-for-houstons-affinity-for-thesexual-perversion-movement/

[2] Swanson K. Hurricane Harvey - What's the Message? America Hit Again and Again and Again. Generations. https://www.generations.org/programs/743 Published 31 August 2017. Accessed 30 September 2017. See also: http://www.rightwingwatch.org/post/kevin-swansonhurricane-harvey-is-gods-judgment-on-houston-for-havinga-very-aggressively-pro-homosexual-mayor/

[3] @ AnnCoulter. I don't believe Hurricane Harvey is God's punishment for Houston electing a lesbian mayor. But that is more credible than "climate change." Twitter. https://twitter.com/AnnCoulter/ status/902373016818126849 Published 28 August 2017. Accessed 30 September 2017.

[4] Steinberg T. Acts of God: The unnatural history of natural disaster in America. Oxford University Press. 2006. ISBN: 9780195309683

[5] Colbert S. Hurricane Isaac at GOP Convention. Comedy Central. http://www.cc.com/video-clips/z0q2d6/the-colbert-reporthurricane-isaac-at-gop-convention Published 28 August 2012. Accessed 30 September 2017.

[6] Geiger T, Frieler K, Levermann A. High-income does not protect against hurricane losses. Env. Res. Letters, 2016;11(8):084012. http: //stacks . iop.org/1748-9326/11/i=8/a=084012

[7] Walworth, Janis. Do Gays Cause Hurricanes? Do "Unnatural" Acts Cause Natural Disasters? Center for Gender Sanity. http://web.archive.org/web/19991004182841/www.cris.com/ merkins/orlando.shtml. Published 16 July 1998. Accessed 30 September 2017.

[8] Burnett D. How same-sex marriage causes floods. The Guardian. https://www.theguardian.com/science/brainflapping/2014/jan/21/how-same-sex-marriage-causes-floods Published 21 January 2014. Accessed 30 September 2017.

[9] Warren R and Olejniczak MJ. Hurricane Strikes as Divine Retribution: An Empirical Test. Skeptic Magazine, 2016;21(1):19-21.

[10] Dobson, J. Marriage Under Fire: Why We Must Win This Battle. Multnomah Publishers, 2004. ISBN: 1590524314

[11] Murphy JD. Storm Data Preparation (National Weather Service Instruction 10-1605), 2016. http://www.nws.noaa.gov/directives/ sym/pd01016005curr.pdf

[12] Gall M, Borden KA, Cutter SL. When do losses count? Six fallacies of natural hazards loss data. Bulletin of the American Meteorological Society, 2009;90(6),799-809. DOI: 10.1175/2008BAMS2721.1

[13] dos Santos RP. Some comments on the reliability of NOAA's Storm Events Database. arXiv, 2016;1606.06973v2 [cs.OH]

[14] US Census Bureau. County intercensal estimates (20002010). https://www . census.gov/data/datasets/time-series/demo/ popest/intercensal-2000-2010-counties.html Published 02 December 2016. Accessed 10 October 2017.

[15] US Census Bureau. State Population Totals Datasets. https://www . census.gov/data/datasets/2016/demo/popest/state-total.html Published 16 December 2016. Accessed 10 October 2017.

[16] R Core Team (2017). R: A language and environment for statistical computing. R Foundation for Statistical Computing, Vienna, Austria. URL https://www.R-project.org/.

[17] RStudio Team (2016). RStudio: Integrated Development for R. RStudio, Inc., Boston, MA. URL http://www.rstudio.com/.
[18] R packages:

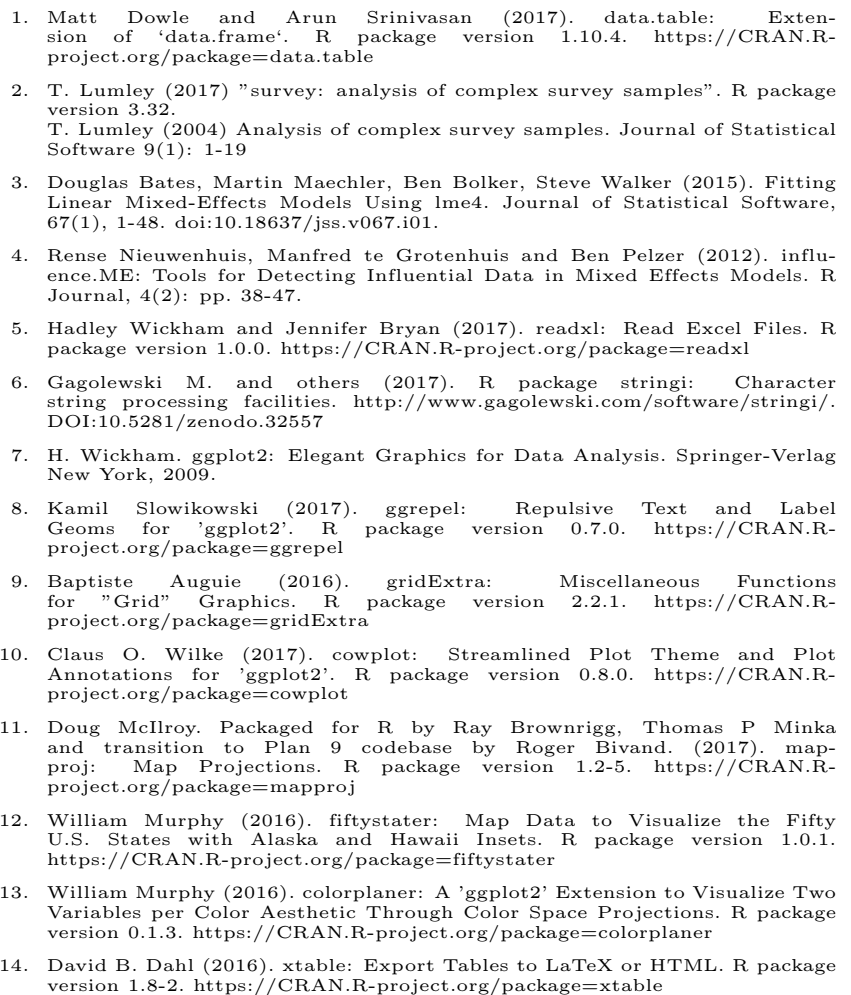

19] Bosco F, Carp J, Field JG, et al. Maximizing the Reproducibility of Your Research. PsyArXiv, 2017. DOI: 10.17605/OSF.IO/K9MN3

[20] Winter B. Linear models and linear mixed effects models in $\mathrm{R}$ with linguistic applications. arXiv, 2013;1308.5499 [cs.CL].

[21] Heavner, J. "Memes in Mathematics: More Than Just a Joke". arXiv, April 12018 [math.HO].

[22] Johnson VE. Revised standards for statistical evidence. PNAS, 2013;110(48):19313-19317. DOI:10.1073/pnas.1313476110

[23] Coleman TA, Dixon PG. An objective analysis of tornado risk in the United States. Weather and Forecasting, 2014;29(2):366-376. DOI 10.1175/WAF-D-13-00057.1

[24] Few S. Dual-Scaled Axes in Graphs - Are They Ever the Best Solution? Visual Business Intelligence Newsletter, March 2008.

[25] Gates GJ. "Demographics of married and unmarried same-sex couples: Analyses of the 2013 American Community Survey." The Williams Institute, 2015. https://williamsinstitute.law. ucla.edu/wp-content/uploads/Demographics-Same-Sex-CouplesACS2013-March-2015.pdf Published March 2015. Accessed 10 October 2017.

[26] Cohen, PN. Homogamy Unmodified. Journal of Family Theory \& Review, 2011;3(1):47-51. 10.1111/j.1756-2589.2010.00080.x

[27] Lofquist DA, Lewis JM. Improving Measurement of Samesex Couples. US Census Bureau. SEHSD Working Paper 2015-13. https://www. census.gov/content/dam/Census/library/ working-papers/2015/demo/SEHSD-WP2015-13.pdf

[28] Croux C, Dehon C. Influence functions of the Spearman and Kendall correlation measures. Statistical methods \& applications, 2010;19(4):497-515. DOI: 10.1007/s10260-010-0142-Z

[29] US Census Bureau. State Area Measurements and Internal Point Coordinates. https://www.census.gov/geo/reference/ state-area.html Published August 2010. Accessed 10 October 2017 


\section{Appendix A: R Code}

This code and all "summary" datafiles may be downloaded from https://github.com/abissellsiders/gays_dont_ cause_storms. This code is genuinely copy-paste-able and may be placed into an R program editor of one's choice. If one does not download the three summary datafiles (acs_summary_dt.rds, sed_summary_dt.rds, pop_dt.rds), then this code will attempt to load the source data from three directories (acs_wd, sed_wd, pop_wd).

If one wishes to examine the ACS data through its processing steps, one can access this data at osf.io/yujdz.

If one wishes to run this code in full, one must use the code documented at https://github.com/abissellsiders/ datafiles under folders "american_community_survey", "storm_events_database", "us_census_population_estimates" in order to download the source dtabases, and one must change the three variables "acs_wd", "sed_wd", "pop_wd" to the appropriate directories.

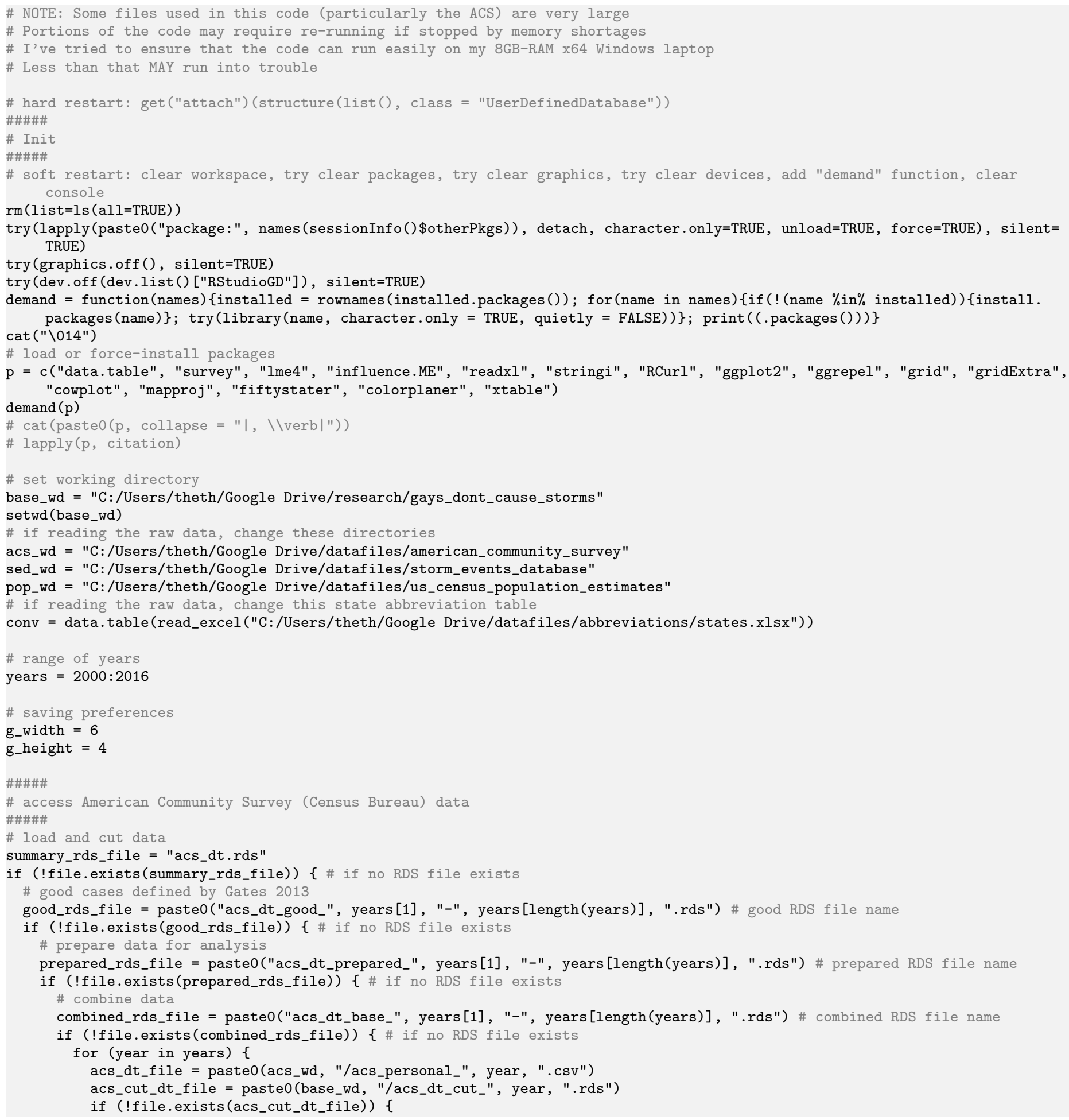




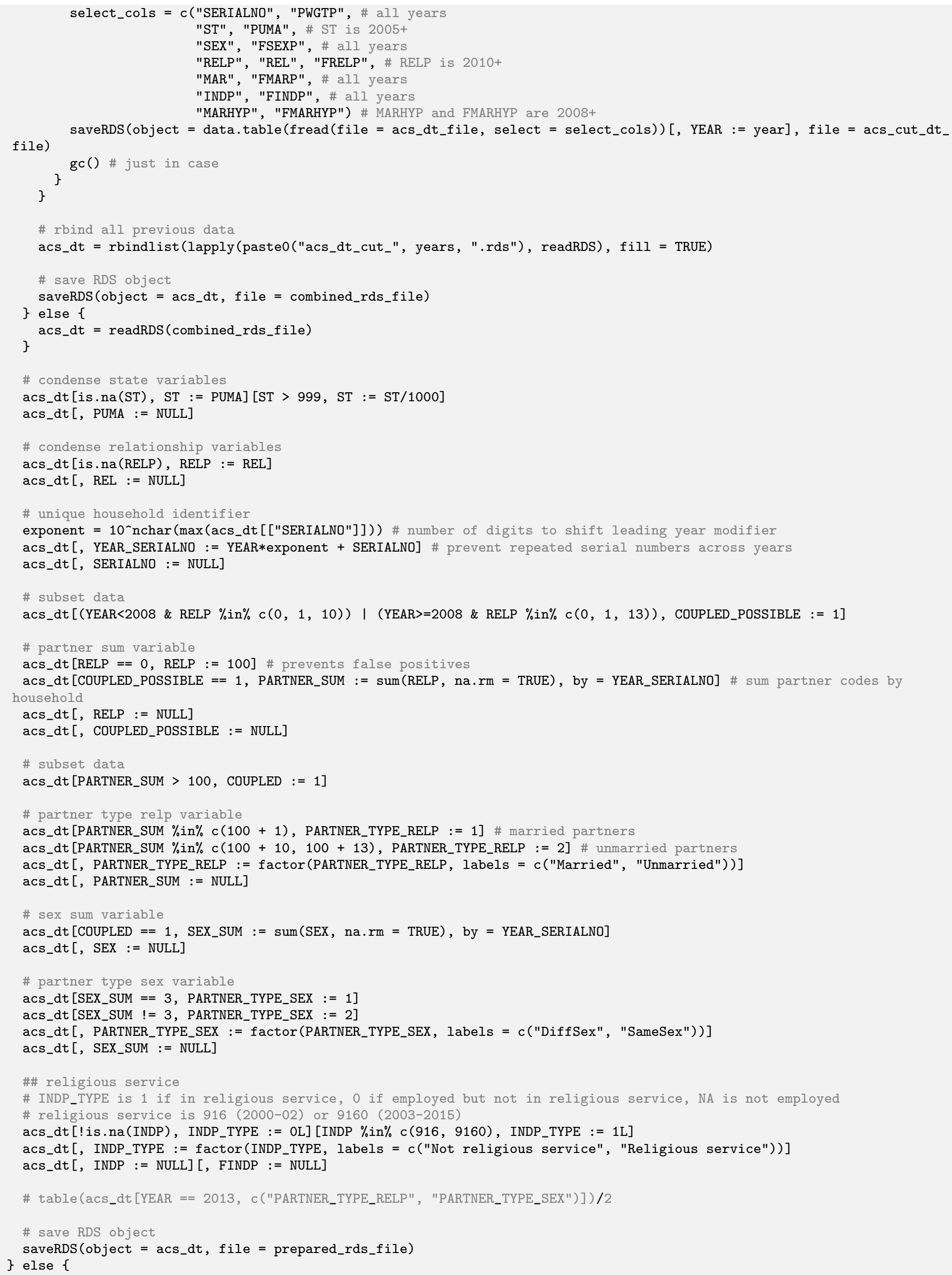


\# \# bad = results with relationship allocation flag

\# acs_dt [, FRELP_SUM $:=\operatorname{sum}($ FRELP, na.rm = TRUE), by = YEAR_SERIALNO $]$

\# acs_dt [FRELP_SUM $>0$, GOOD :=0 ]

\# acs_dt $[$, FRELP := NULL $][$, FRELP_SUM := NULL $]$

\# bad = results with relationship allocation flag

acs_dt [COUPLED $==1$, FMARP_SUM $:=\operatorname{sum}($ FMARP, na.rm $=$ TRUE), by = YEAR_SERIALNO]

acs_dt [FMARP_SUM $>0$, GOOD $:=0]$

acs_dt $[$, FMARP $:=$ NULL $][$, FMARP_SUM $:=$ NULL $]$

\# $\mathrm{bad}=$ results with sex allocation flag

acs_dt [COUPLED $==1$, FSEXP_SUM $:=\operatorname{sum}(F S E X P$, na.rm $=$ TRUE), by = YEAR_SERIALNO]

acs_dt [FSEXP_SUM $>0$, GOOD $:=0]$

acs_dt $[$, FSEXP $:=$ NULL $][$, FSEXP_SUM $:=$ NULL $]$

\# bad = results with marriage year allocation

acs_dt [COUPLED $==1$, FMARHYP_SUM $:=$ sum(FMARHYP, na.rm $=$ TRUE), by $=$ YEAR_SERIALNO]

acs_dt[PARTNER_TYPE_RELP $==$ "Married" \& FMARHYP_SUM $>0$, GOOD $:=0]$

acs_dt [, FMARHYP $:=$ NULL] [, FMARHYP_SUM $:=$ NULL]

acs_dt [COUPLED $==1$, MARHYP_SUM $:=\operatorname{sum}($ MARHYP), by $=$ YEAR_SERIALNO]

\# bad = results with different years

\# removes 0 entires

\# acs_dt [PARTNER_TYPE_RELP $==$ "Married" \& MARHYP_SUM $!=$ MARHYP $* 2$, GOOD :=0]

\# bad = results with same-sex marriage before 2004

acs_dt[PARTNER_TYPE_RELP $==$ "Married" \& MARHYP_SUM $==$ MARHYP $* 2$ \& PARTNER_TYPE_SEX $==$ "SameSex" \& MARHYP < 2004, GOOD := 0]

acs_dt $[$, MARHYP := NULL] [, MARHYP_SUM := NULL]

\# compare results to gates 2013

t_all $=$ table $($ acs_dt $[$ YEAR $==2013$, c ("PARTNER_TYPE_RELP", "PARTNER_TYPE_SEX" $)]) / 2$

$t_{-}$good $=\operatorname{table}($ acs_dt $[Y E A R==2013 \&$ GOOD $==1$, c ("PARTNER_TYPE_RELP", "PARTNER_TYPE_SEX")] $) / 2$

t_all - t_good

gates_method_dt $=a c s_{-} d t[G O O D==1],[, .($ PWGTP_SUM $=$ sum $($ PWGTP $))$, by $=$ c ("YEAR", "PARTNER_TYPE_SEX", "PARTNER_TYPE_RELP" $)$ ] [, METHOD := "Gates"]

naive_method_dt $=a c s \_d t[$ ! is.na (PARTNER_TYPE_SEX) \& ! is.na (PARTNER_TYPE_RELP), . (PWGTP_SUM = sum (PWGTP)), by = c ("YEAR", "

PARTNER_TYPE_SEX", "PARTNER_TYPE_RELP")] [, METHOD := "Naive"]

method_dt $=$ rbind (gates_method_dt, naive_method_dt)

ggplot (method_dt, aes $(x=$ YEAR, $y=$ PWGTP_SUM, color $=$ METHOD $))+$

geom_point () + geom_line() +

facet_grid(PARTNER_TYPE_SEX PARTNER_TYPE_RELP, scales = "free_y")

ggsave ("fig_appendix_c_methods_count.png", width = g_width, height = g_height)

gates_proportion_dt $=$ copy (gates_method_dt) $[$, PWGTP_SUM_SUM $:=$ sum (PWGTP_SUM), by = YEAR] [PARTNER_TYPE_SEX == "SameSex",

] [, . (PROPORTION_SAME_SEX $=\operatorname{sum}($ PWGTP_SUM / PWGTP_SUM_SUM)), by = c ("YEAR") ] [, METHOD := "Gates"]

naive_proportion_dt $=$ copy (naive_method_dt) [, PWGTP_SUM_SUM $:=$ sum (PWGTP_SUM), by = YEAR] [PARTNER_TYPE_SEX == "SameSex",

] [, . (PROPORTION_SAME_SEX $=\operatorname{sum}($ PWGTP_SUM / PWGTP_SUM_SUM)), by = c ("YEAR") ] [, METHOD := "Naive"]

proportion_dt = rbind (gates_proportion_dt, naive_proportion_dt)

ggplot (proportion_dt, aes $(x=$ YEAR, y = PROPORTION_SAME_SEX, color $=$ METHOD) ) +

geom_point() + geom_line()

ggsave("fig_appendix_c_methods_proportion.png", width = g_width, height = g_height)

\# save RDS object

saveRDS (object $=$ acs_dt, file = good_rds_file)

\} else \{

\} acs_dt $=$ readRDS $($ good_rds_file $)$

\#\# summary statistics

acs_summarizer_sex = function(year) \{

coup_design $=$ svydesign $($ ids $=\sim 0$, \# no strata

weights $=\sim$ PWGTP, \# weight variable

data $=$ acs_dt $[$ YEAR $==$ year \& GOOD $==1]$,

coup_dt $=$ data $\cdot$ table $($ svyby $($ formula $=\sim$ PARTNER_TYPE_SEX,

by $=\sim \mathrm{ST}$,

design = coup_design,

FUN = svymean, 


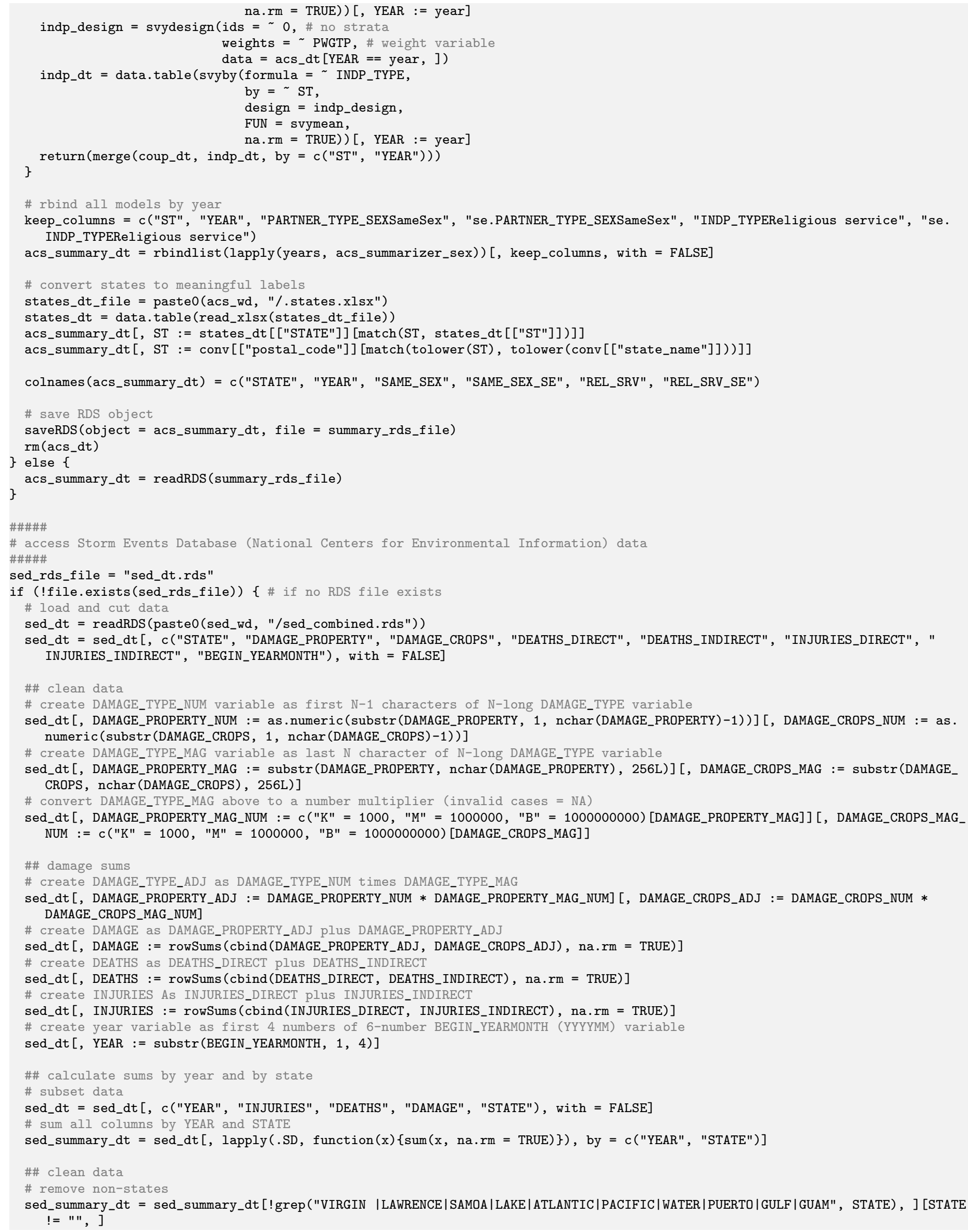


\# convert STATE field to characters

sed_summary_dt [, STATE := as.character(STATE)]

\# convert STATE field to abbreviations

sed_summary_dt[, STATE := conv[["postal_code"]][match(tolower(STATE), tolower(conv[["state_name"]]))]]

\# convert YEAR field to numeric

sed_summary_dt [, YEAR := as.numeric(YEAR)]

\# states with no damage reported probably received a very small amount of damage (and log scale can't handle zero values);

the minimum present in other states and other years is 1,000

sed_summary_dt [DAMAGE $==0$, DAMAGE $:=1000]$

\# save to RDS

saveRDS (object $=$ sed_summary_dt, file $=$ sed_rds_file)

\} else \{ \# load the RDS file

\}

sed_summary_dt $=$ readRDS $($ sed_rds_file)

\#\#\#\#\#

\# access population estimates (Census Bureau) data

\#\#\#\#

\# load data

pop_rds_file = "pop_dt.rds"

if (!file.exists (pop_rds_file)) \{

pop_dt $=\operatorname{readRDS}($ paste0 $($ pop_wd, "/pop_dt.rds"))

\# convert STATE field to abbreviation

pop_dt [, STATE := conv[["postal_code"]][match(tolower(STATE), tolower(conv[["state_name"]]))]]

\# create state size variable

pop_dt [, SIZE := as.numeric(conv[["area_square_miles"]][match(STATE, conv[["postal_code"]])])]

\# save to RDS

saveRDS (object $=$ pop_dt, file $=$ pop_rds_file)

\} else $\{$ \# load the RDS file

\}

pop_dt $=$ readRDS $\left(p o p \_r d s \_f i l e\right)$

\#\#\#\#

\# merge data

\#\#\#\#\#

\# merge data sideways

all_dt $=\operatorname{Reduce}(f \operatorname{unction}(\ldots)\{\operatorname{merge}(\ldots$, by $=c($ "STATE", "YEAR")), list (sed_summary_dt, acs_summary_dt, pop_dt))

\# create data for the united states as a whole

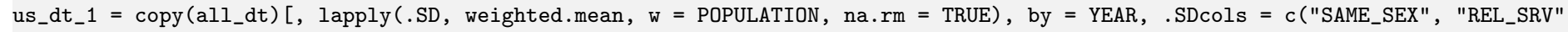
)]

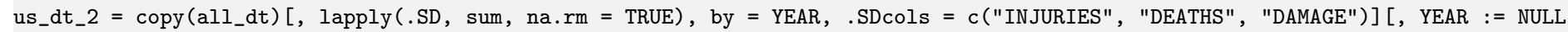
]

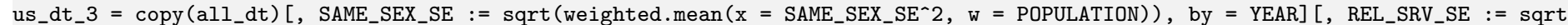
(weighted.mean $(\mathrm{x}=$ REL_SRV_SE^ 2 , w $=$ POPULATION)), by = YEAR $[$, POPULATION $:=$ sum (POPULATION), by $=$ YEAR $[$, c ("SAME_SEX_SE ", "REL_SRV_SE", "POPULATION"), with = FALSE]

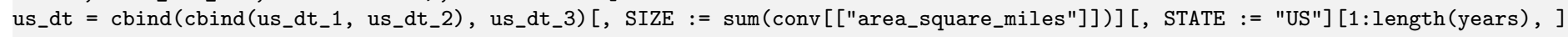

\# rbind datasets

all_dt $=$ rbind $\left(a l l_{-} d t\right.$, us_dt $)$

\# calculate per capita

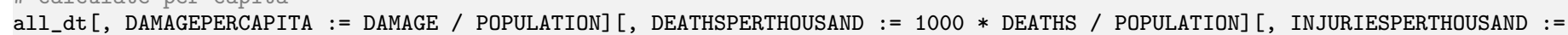
$1000 *$ INJURIES / POPULATION] [, POPDENSITY := POPULATION / SIZE]

\# make states into factors

all_dt $[$, STATE := factor(STATE) $]$

\# relevant data only

all_dt_rel = all_dt [! (STATE \%in\% c ("US")), ]

all_dt_rel[, SAME_SEX $:=$ SAME_SEX $*$ 100] [, REL_SRV $:=$ REL_SRV * 100]

all_df_rel = data.frame (all_dt_rel)

\#\#\#\#\#

\# Kendall's rank correlation

\#\#\#\#\#

\# significance codes

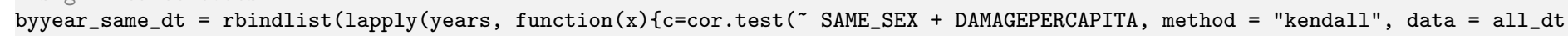
_rel [YEAR $==x],) ; \operatorname{return}($ data.table $($ year $=x$, tau_same $=c[$ "estimate"], p_same $=c[" p . v a l u e "]))\})$ ) [p_same < 0.05, sig_ same $:=" * "]\left[p_{-}\right.$same $<0.01$, sig_same $\left.:=" * * "\right]\left[p_{-}\right.$same $<0.001$, sig_same := "***"]

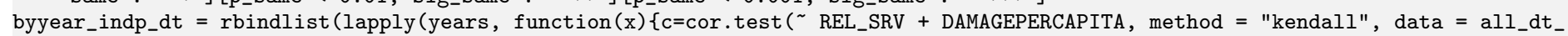

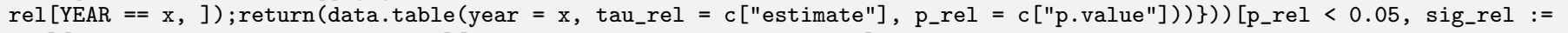
"*"] [p_rel < 0.01, sig_rel := "**"] [p_rel < 0.001 , sig_rel := "***"]

\# merge data 
\# print table

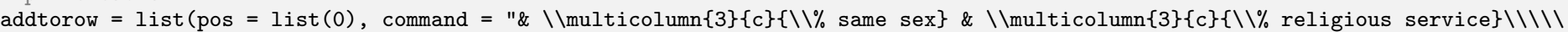

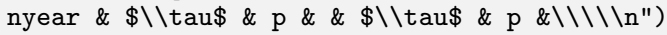

$\mathrm{tab}=$ xtable (byyear_merge_dt, type = "latex", caption = "Kendall's rank correlation results", digits = c (1,4,2,5,1,2,5,1)) align $(t a b)=$ "lr|rrl|rrl"

print (tab,

file = "fig_kendall.tex", add.to.row = addtorow, include. rownames $=$ FALSE, include. colnames $=$ FALSE, caption.placement = "bottom", floating.environment = "figure")

\#\#\#\#\#

\# Mixed linear regression

\#\#\#\#\#

\# data is approximately normal

qqnorm(log10(all_dt_rel[["DAMAGEPERCAPITA"]]))

qqnorm (all_dt_rel[["SAME_SEX"]])

qqnorm (all_dt_rel[["REL_SRV"]])

qqnorm (log10(all_dt_rel[["POPDENSITY"] ]))

\# linear regressions

lcontrol = lmerControl (optCtrl = list $(\operatorname{maxfun}=1 \mathrm{e} 6))$

lmer_same_null $=\operatorname{lmer}(\log 10($ DAMAGEPERCAPITA $) \sim(1+$ SAME_SEX $\mid$ STATE $)+(1+$ SAME_SEX $\mid$ YEAR $)$, data $=$ all_df_rel, weights = POPULATION/mean(POPULATION), REML = FALSE, control = lcontrol)

lmer_same_full $=\operatorname{lmer}(\log 10($ DAMAGEPERCAPITA $) \sim$ SAME_SEX + $(1+$ SAME_SEX $\mid$ STATE $)+(1+$ SAME_SEX $\mid$ YEAR $)$, data = all_df_rel, weights $=$ POPULATION/mean (POPULATION), REML = FALSE, control $=1$ control)

lmer_indp_null $=\operatorname{lmer}(\log 10($ DAMAGEPERCAPITA $) \sim(1+$ REL_SRV|STATE $)+(1+$ REL_SRV|YEAR $)$, data = all_df_rel, weights = POPULATION/mean (POPULATION), REML = FALSE, control = lcontrol)

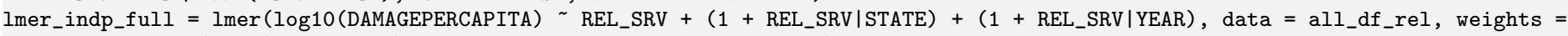
POPULATION/mean (POPULATION), REML = FALSE, control = lcontrol)

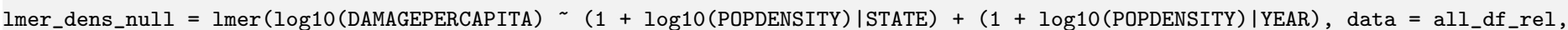
weights $=$ POPULATION $/$ mean (POPULATION), REML = FALSE, control = lcontrol)

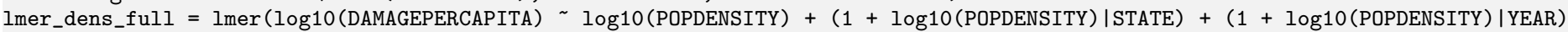
, data $=$ all_df_rel, weights $=$ POPULATION $/$ mean (POPULATION), REML = FALSE, control = lcontrol)

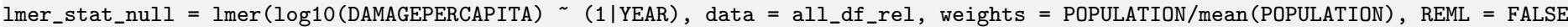
, control = lcontrol)

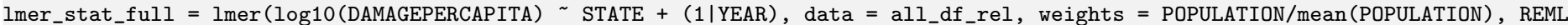
= FALSE, control = lcontrol)

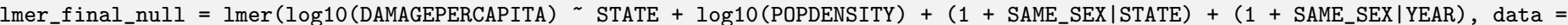
all_df_rel, weights = POPULATION/mean (POPULATION), REML = FALSE, control = lcontrol)

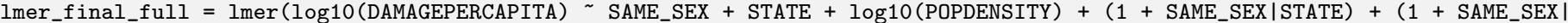

YEAR), data $=$ all_df_rel, weights $=$ POPULATION/mean (POPULATION), REML = FALSE, control = lcontrol)

anova(lmer_final_null, lmer_final_full)

lmer_diagnostics = function(lmer_model, func_output_filename) \{

func_residuals = residuals (lmer_model, type = "pearson")

func_fitted $=$ fitted (lmer_model)

\# residuals vs fitted

ggplot ()$+\operatorname{aes}(x=$ func_fitted, $y=$ func_residuals $)+$

geom_point ()$+$ geom_hline (yintercept $=0$, color $=$ "red", size $=0.75)+$ geom_smooth (method $=$ "loess") +

scale_x_continuous (sec.axis = dup_axis ()$)+$ scale_y_continuous (sec.axis = dup_axis ()$)+$ theme_minimal ()$+$

labs (title = paste0 (func_output_filename), $x=$ "fitted values", $y=$ "standardized residuals (pearson)")

ggsave (paste0 ("fig_appendix_c_residuals_fitted_", func_output_filename, ".png"), width = g_width, height = g_height)

\# normal q-q

func_quantiles = qqnorm(func_residuals, plot.it = FALSE) $[[1]]$

probs $=c(0.25,0.75)$

$\mathrm{y}=$ quantile (func_residuals, probs, names $=$ FALSE, type $=7$, na.rm $=$ TRUE)

$\mathrm{x}=$ qnorm (probs)

slope $=\operatorname{diff}(y) / \operatorname{diff}(x)$

intercept $=\mathrm{y}[1 \mathrm{~L}]-$ slope $* \mathrm{x}[1 \mathrm{~L}]$

ggplot ()$+\operatorname{aes}(x=$ func_quantiles, $y=$ func_residuals $)+$

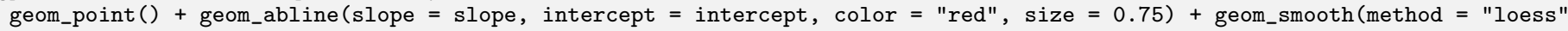
, size $=0.5)+$

scale_x_continuous (sec.axis $=$ dup_axis ()$)+$ scale_y_continuous $(\sec$. axis $=$ dup_axis ()$)+$ theme_minimal ()$+$

labs(title = paste0(func_output_filename), $\mathrm{x}=$ "theoretical quantiles", $y=$ "standardized residuals (pearson)") 


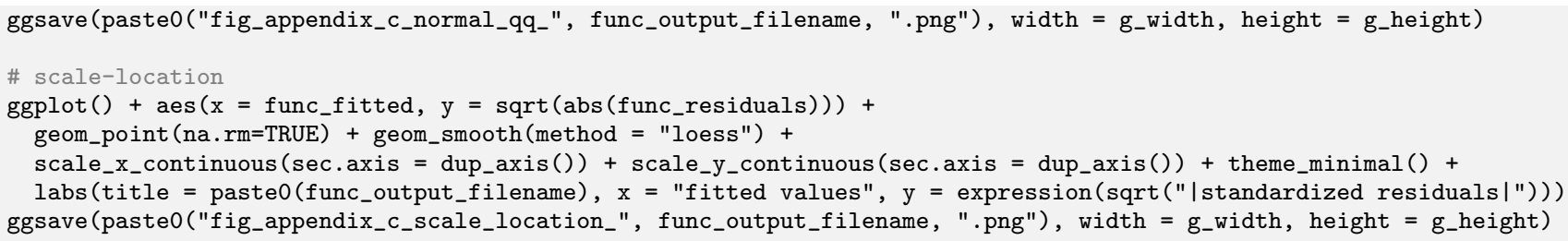

tab $=$ xtable(lmer_dt $[\operatorname{var}==$ "Density", ], type = "latex", caption = "Linear mixed-effects model results", digits = c $(1,1,2,2,0,0,1,5,1))$

align $(t a b)=$ "rrrr|rrrrr"

print (tab,

file = "fig_density.tex",

add.to.row = addtorow,

include. rownames = FALSE,

include. colnames $=$ FALSE,

caption.placement = "bottom",

floating.environment = "figure",

table.placement $=$ "H") 


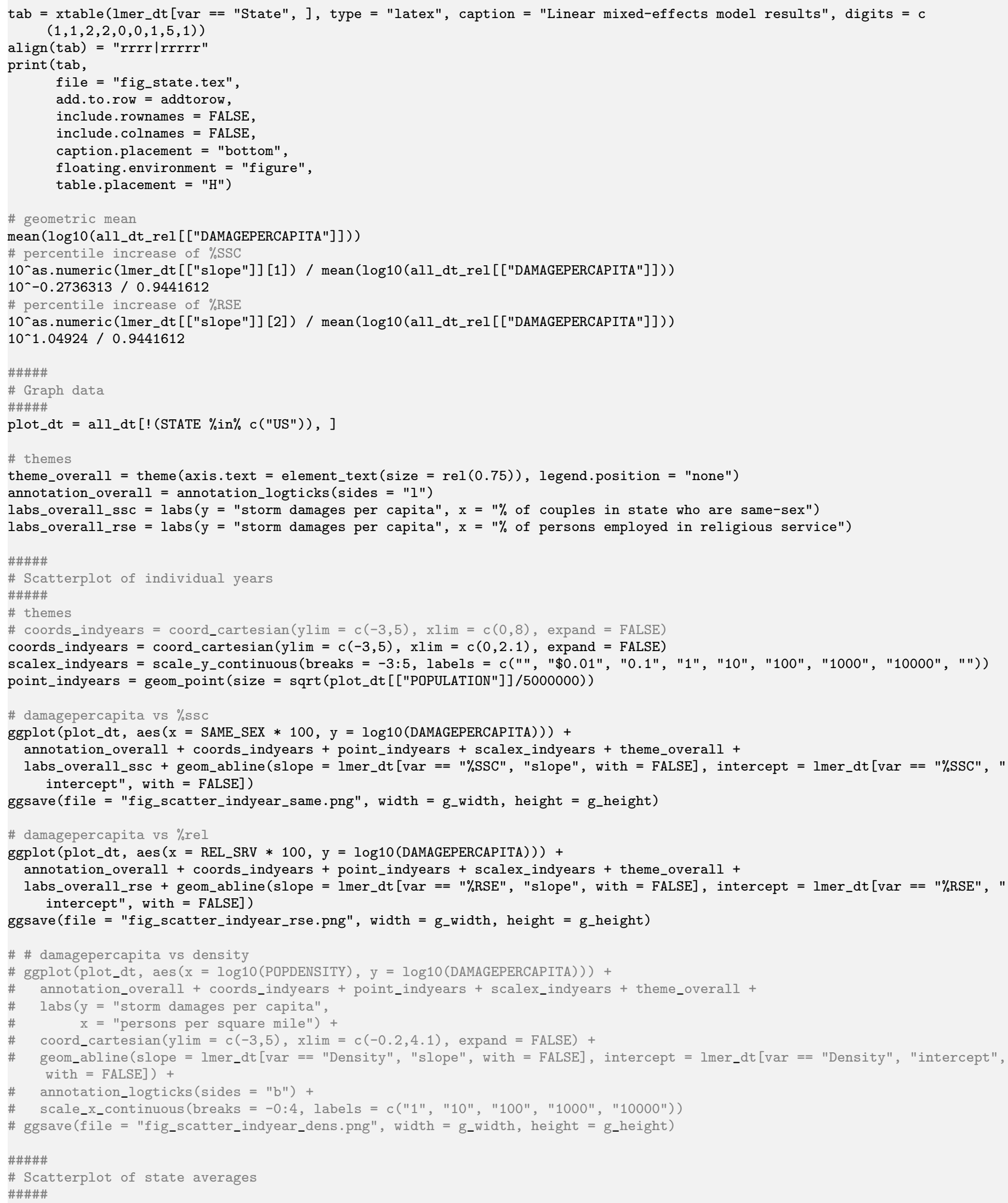




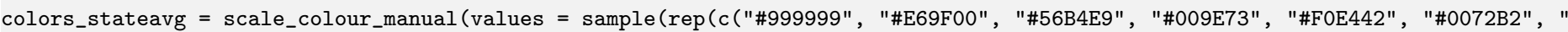
\#D55E00", "\#CC79A7"), len = 51)))

coords_stateavg $=\operatorname{coord}$ _cartesian $(y \lim =c(0.3,3.0), x l i m=c(0.1,1.3)$, expand $=$ FALSE $)$

scalex_stateavg $=$ scale_y_continuous $($ breaks $=c(1,2,3)$, labels $=c(" \$ 10 ", " 100 ", " 1000 "))$

\# damagepercapita vs \%ssc

ggplot (copy(state_dt) [STATE $!=$ "US", ] [STATE $==$ "DC", STATE $:=$ "DC"], aes $(x=$ SAME_SEX $* 100$, color $=$ STATE, y = log10( DAMAGEPERCAPITA)) +

geom_point ()$+$ geom_text_repel (aes $(l$ abel $=$ STATE $), \min$. segment.length $=0$, box.padding $=0.1)+$

labs_overall_ssc + annotation_overall + colors_stateavg + coords_stateavg + scalex_stateavg + theme_overall

ggsave(file = "fig_scatter_average_same.png", width = g_width, height = g_height)

\# damagepercapita vs $\%$ rel

ggplot (state_dt[STATE ! = "US", ], aes $(\mathrm{x}=$ REL_SRV $* 100$, color = STATE, y = log10(DAMAGEPERCAPITA)) +

geom_point ()$+$ geom_text_repel (aes $($ label $=$ STATE), min. segment.length $=0$, box $\cdot$ padding $=0.1)+$

labs_overall_rse + annotation_overall + colors_stateavg + coords_stateavg + scalex_stateavg + theme_overall ggsave(file = "fig_scatter_average_rse.png", width = g_width, height = g_height)

\#\#\#\#

\# Chloropleth state map

\#\#\#\#\#

$\mathrm{g}=\operatorname{ggplot}(\operatorname{copy}($ state_dt) $[$, STATE := tolower(conv[["state_name"]] [match(STATE, conv[["postal_code"]])])] [! (STATE \%in\% c(" district of columbia", "united states")), ], aes(map_id = STATE, fill = REL_SRV * 100, fill2 = SAME_SEX $* 100$, label = SAME_SEX * 100)) +

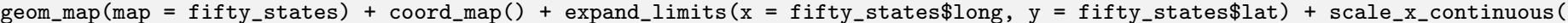
breaks $=$ NULL $)+$ scale_y_continuous $($ breaks $=$ NULL $)+1$ abs $(x=" ", y=" 1)+$

scale_fill_colorplane(axis_title = $" \%$ religious jobs", axis_title_y = $\%$ same-sex couples", breaks = seq( 0.5 , 1.25 , by = $0.25)$, breaks_y $=\operatorname{seq}(0.75,1.25$, by $=0.25)$, color_projection = red_blue_projection) + guides $(f i l l=$ guide_colorplane ( title = "State Color Key")) +

theme_classic ()$+$ theme(legend.position $=c(0.875,0.125)$, legend.background $=$ element_blank())

ggsave(file = "fig_chloropleth_same_rse.png", width = g_width*1.5, height = g_height*1.5)

\#\#\#\#\#

\# Lineplot each state damage and year

\#\#\#\#\#

\# melt data

melt_dt $=\operatorname{melt}\left(a l l_{-} d t, i d . v a r s=c(" S T A T E "\right.$, "YEAR"))

melt_dt [, STATE := factor (STATE, levels = conv[["postal_code"]])]

\# melt averages data

line_dt $=\operatorname{melt}($ state_dt, id.vars = c("STATE")) [variable \%in\% c("SAME_SEX", "REL_SRV", "DAMAGEPERCAPITA"), ]

line_dt [, STATE := factor(STATE, levels = conv[["postal_code"]])]

$\mathrm{t}=$ theme (axis.line $=$ element_blank () ,

axis.title.x = element_blank () ,

axis.title.y = element_blank (),

axis.text.y $=$ element_text $(\operatorname{size}=\operatorname{rel}(0.9))$,

strip.background = element_rect (colour="white", fill="white"),

legend.position = "none",

plot.margin $=\operatorname{unit}(\mathrm{c}(0,0,0,1), " \mathrm{~cm} ")$,

panel.grid.major $\mathrm{x}=$ element_line $(\operatorname{color}=$ "gray80", size $=0.5$, linetype $=$ "dotted"))

$1=$ labeller (variable $=c("$ SAME_SEX" = "\%SC", "REL_SRV" = "\%RE", "DAMAGEPERCAPITA" = "damage"), state = function $(\mathrm{x})\{\mathrm{x}\})$

$\mathrm{s}=$ scale_x_continuous (breaks $=c(2000,2005,2010,2015)$, labels $=c(" 2000 ", " ", " 2010 ", " ")$ )

state_g_maker $=$ function $(\mathrm{x})$

states $=\operatorname{conv}[[$ "postal_code" $]][1: 13+13 *(x-1)]$

\# print ("DAMAGEPERCAPITA")

$\mathrm{p} 1=\operatorname{ggplot}()+\mathrm{t}+\mathrm{s}+$

geom_hline (data $=$ line_dt [variable == "DAMAGEPERCAPITA" \& STATE \%in\% states, ], aes $(y$ intercept = log10 (value) ), color = "

lightsalmon1", size $=0.5$, linetype $=$ "solid") +

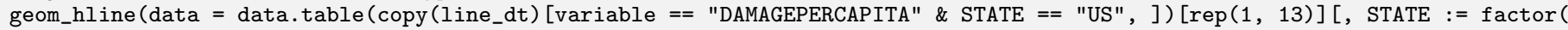

states)], aes $($ yintercept $=\log 10($ value $))$, color $=$ "grey40", size $=0.5$, linetype = "solid") +

geom_line (data $=$ melt_dt [variable $==$ "DAMAGEPERCAPITA" \& STATE \%in $\%$ states \& ! is.na(value), ], aes $(\mathrm{x}=$ YEAR, y $=$ log10(

value)), color $=$ "red") +

facet_grid(variable STATE, scales = "free_y", labeller $=1)+$

coord_cartesian $(y \lim =c(-2,4)$, expand $=$ TRUE $)+$

scale_y_continuous (breaks $=-1: 3$, labels $=c(" \$ 0.1 "$, "1", "10", "100", "1000")) +

theme (axis.text. $\mathrm{x}=$ element_blank () )

\# print ("SAME_SEX")

$\mathrm{p} 2=\operatorname{ggplot}()+\mathrm{t}+\mathrm{s}+$

geom_hline (data $=$ line_dt [variable == "SAME_SEX" \& STATE \%in\% states, ], aes (yintercept = value * 100), color = "

lightsteelblue2", size $=0.5$, linetype = "solid") + 


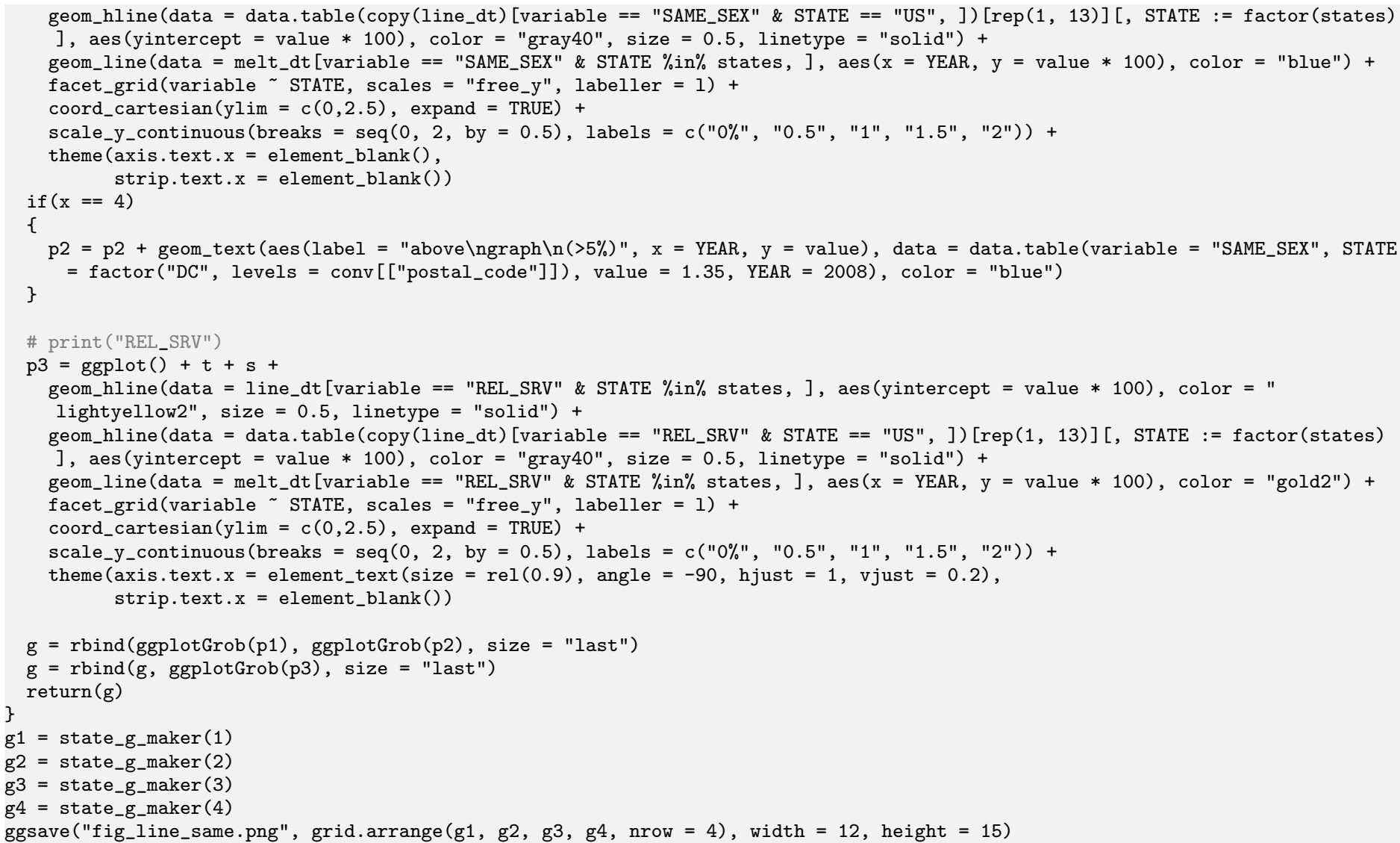


10 Appendix B: Alternative Hypothesis

\section{When God tries to punish your city for} homosexuality but gays use their magic shield to protect it

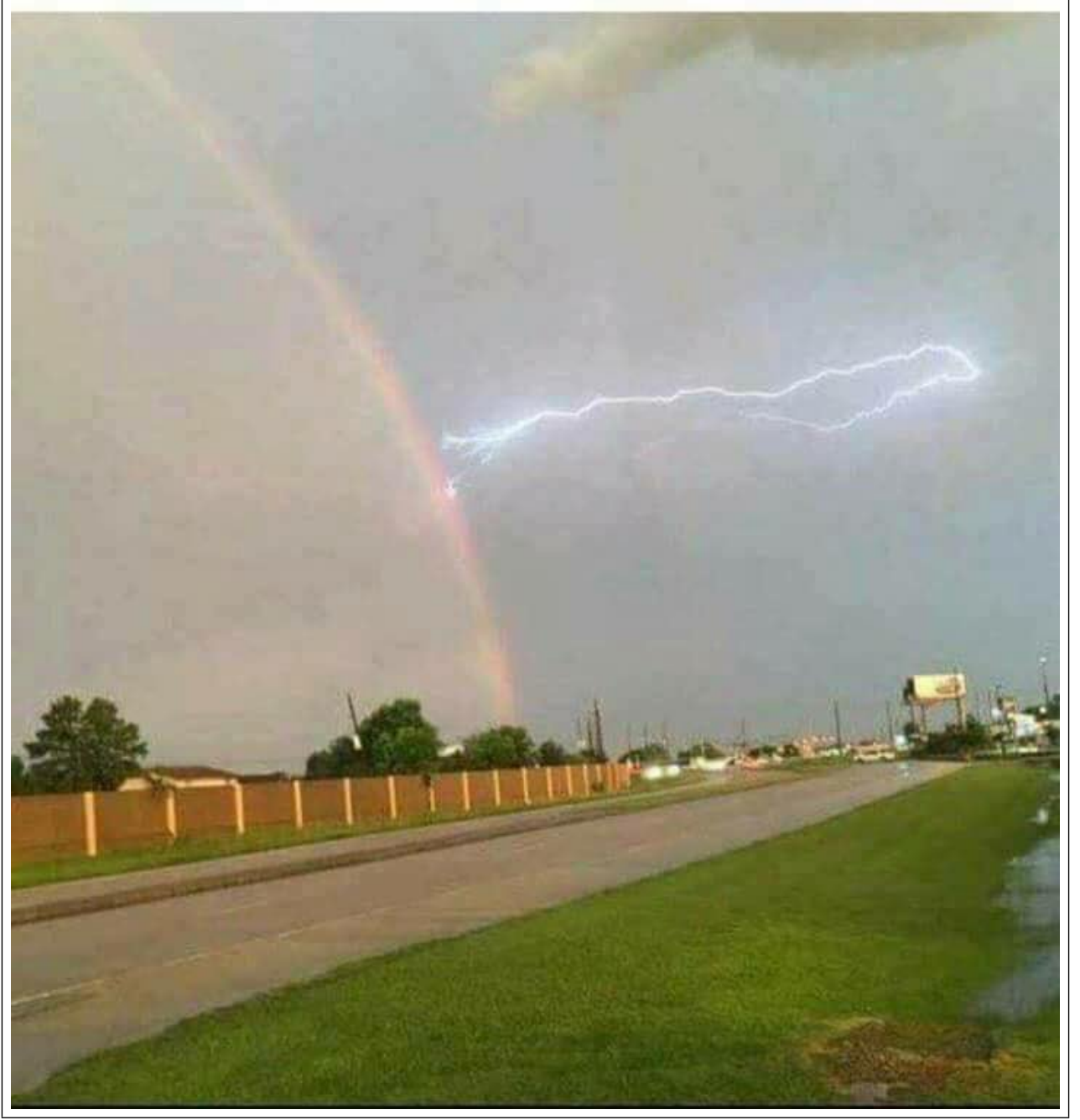




\section{Appendix C: Supplementary Figures}

The naive trend in same-sex couples (count and proportion) would absurdly imply that, in 2008, about 300,000 same-sex couples spontaneously disappeared. Applying Gates 2015's methods produces a trend that is more consistent with reality: steadily increasing numbers of same-sex couples until 2013, when the Census began to recognize same-sex married couples.
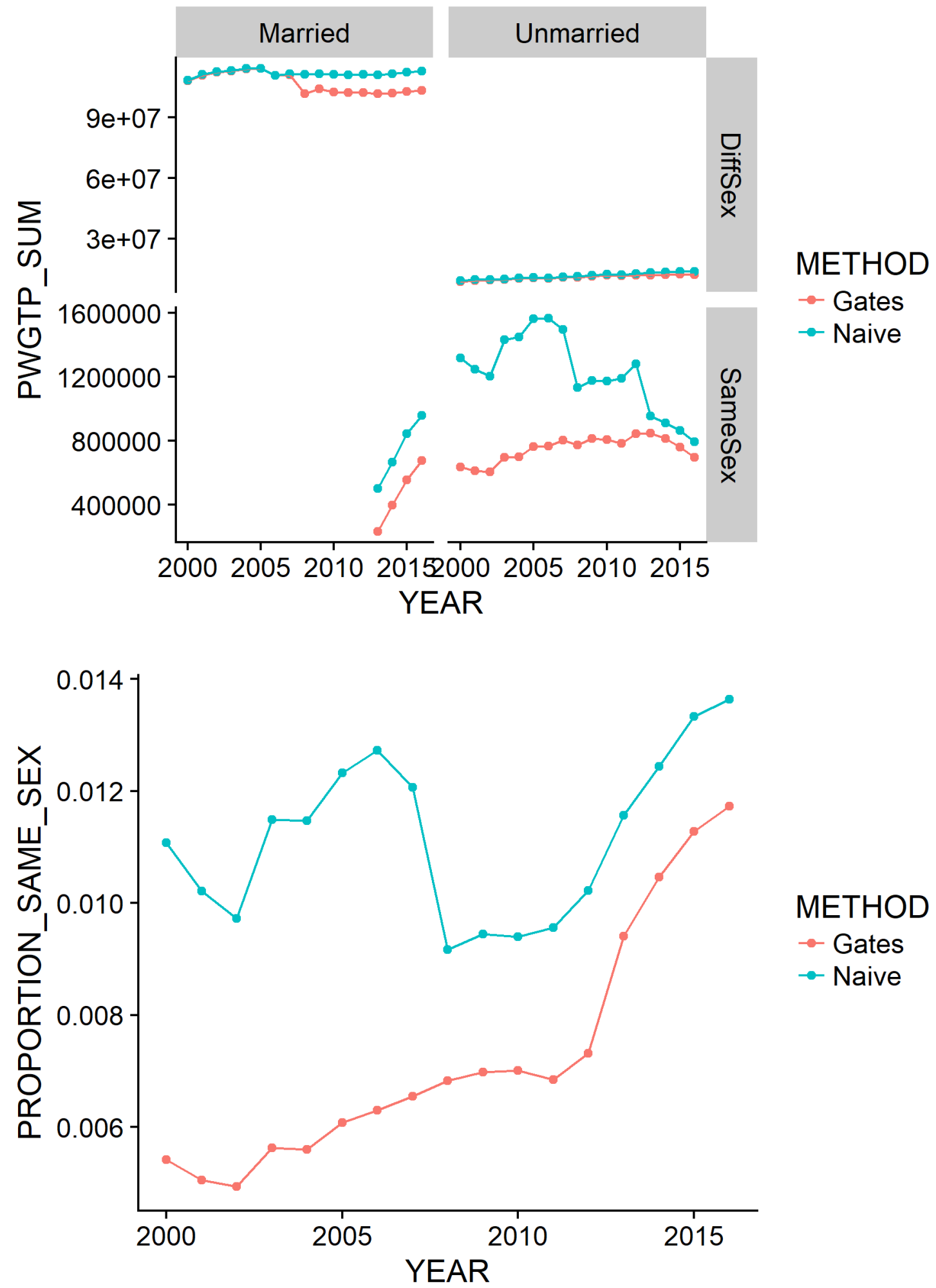
Here are the results of linear mixed-model regression on density and storm damages (area data in square miles obtained from the Census State Area Measurements and Internal Point Coordinates database 29]) (note that this model does not converge, probably because state population density is both [1] non-normal and [2] varies very little by year but is instead a function mostly of the state size and very, very gradual population change):

\begin{tabular}{rrr|rrrrr}
\hline & slope & int & AIC & BIC & $\chi^{2}$ & $\mathrm{p}$ & \\
\hline Density & -0.26 & 1.50 & 2216 & 2258 & 4.0 & 0.04601 & $*$ \\
\hline
\end{tabular}

Figure 11: Linear mixed-effects model results

Population density vs damage/capita in each US state and DC, 2000-16

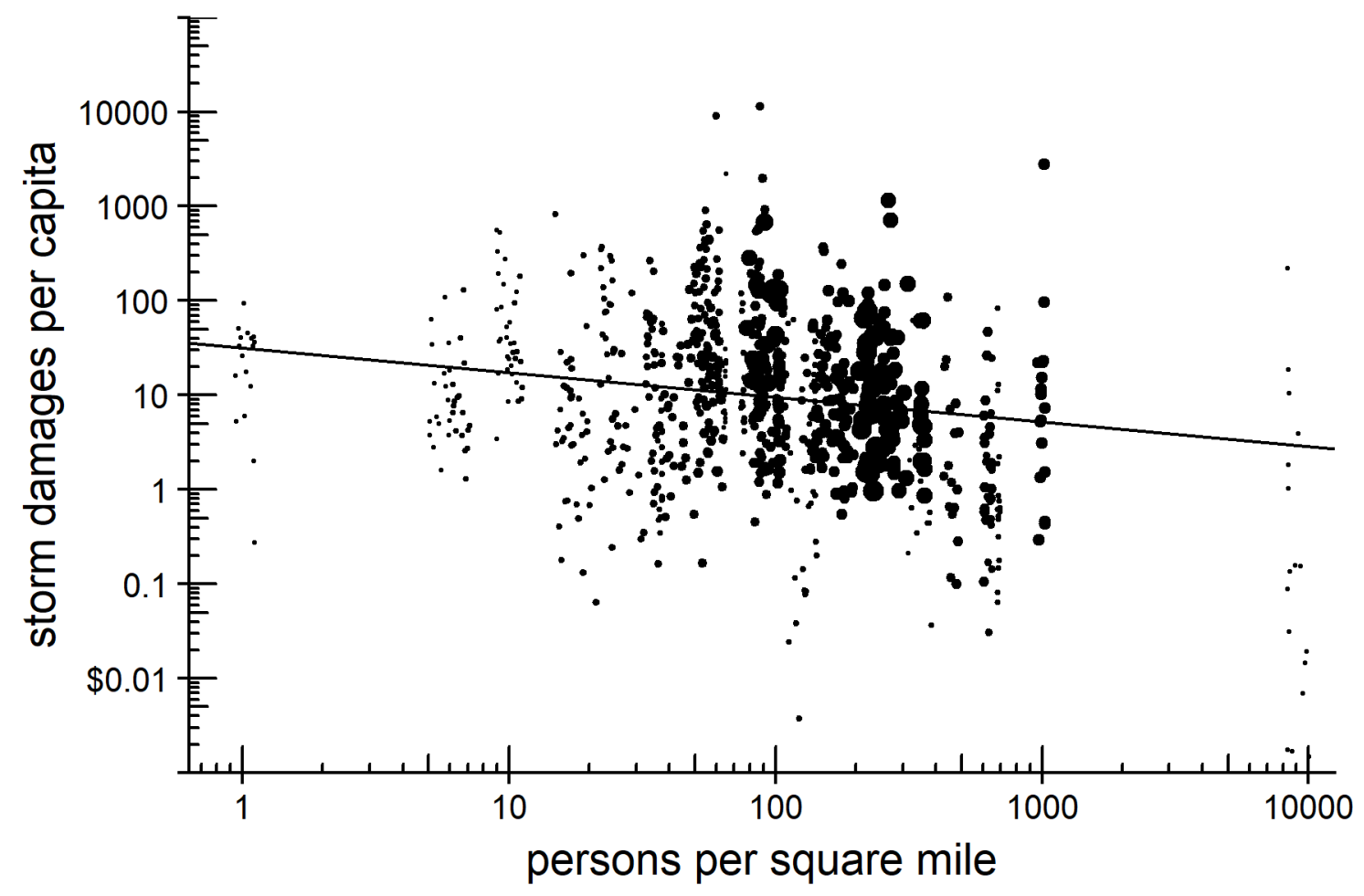


Fitted vs residual values (same-sex, religious service, density, state):

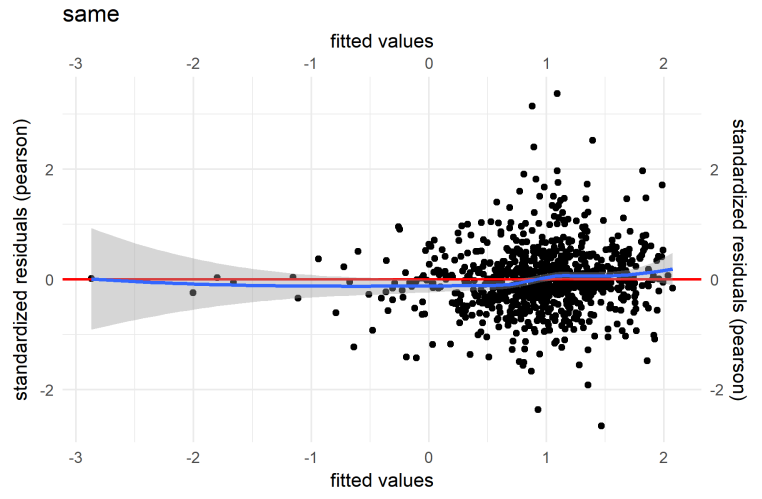

fitted values

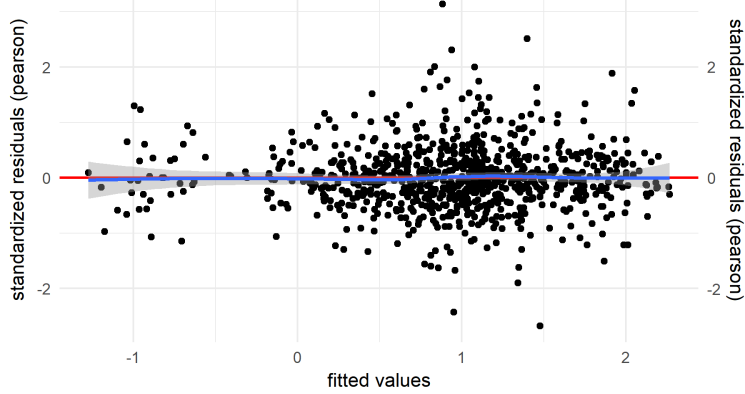

Scale vs location (same-sex, religious service, density, state):
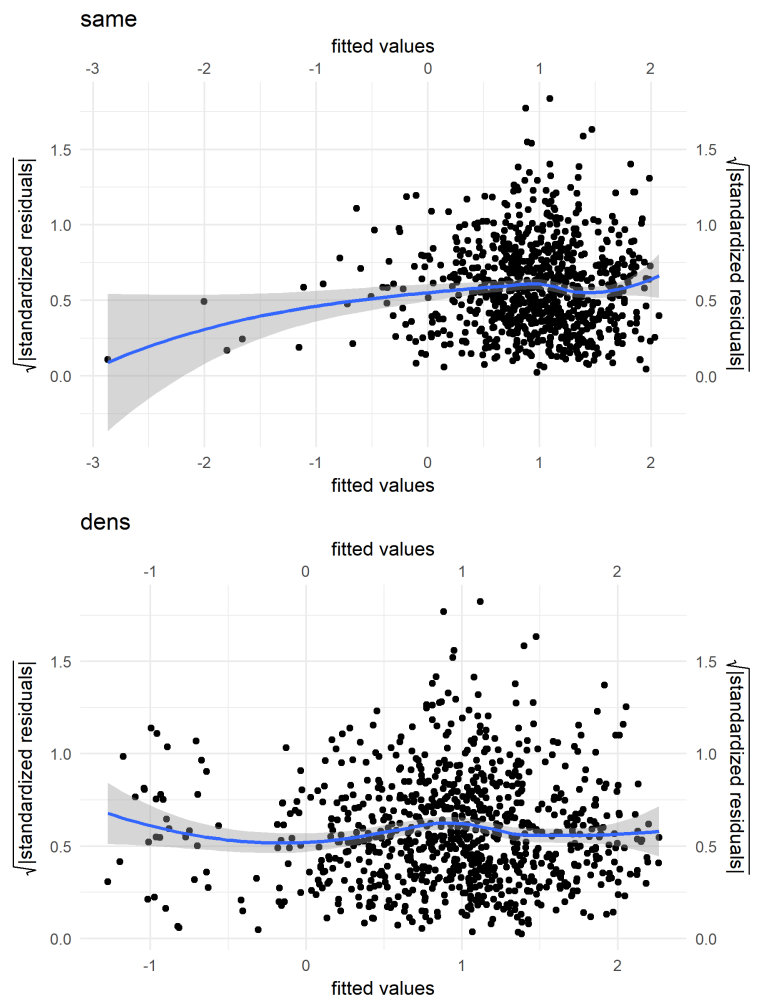
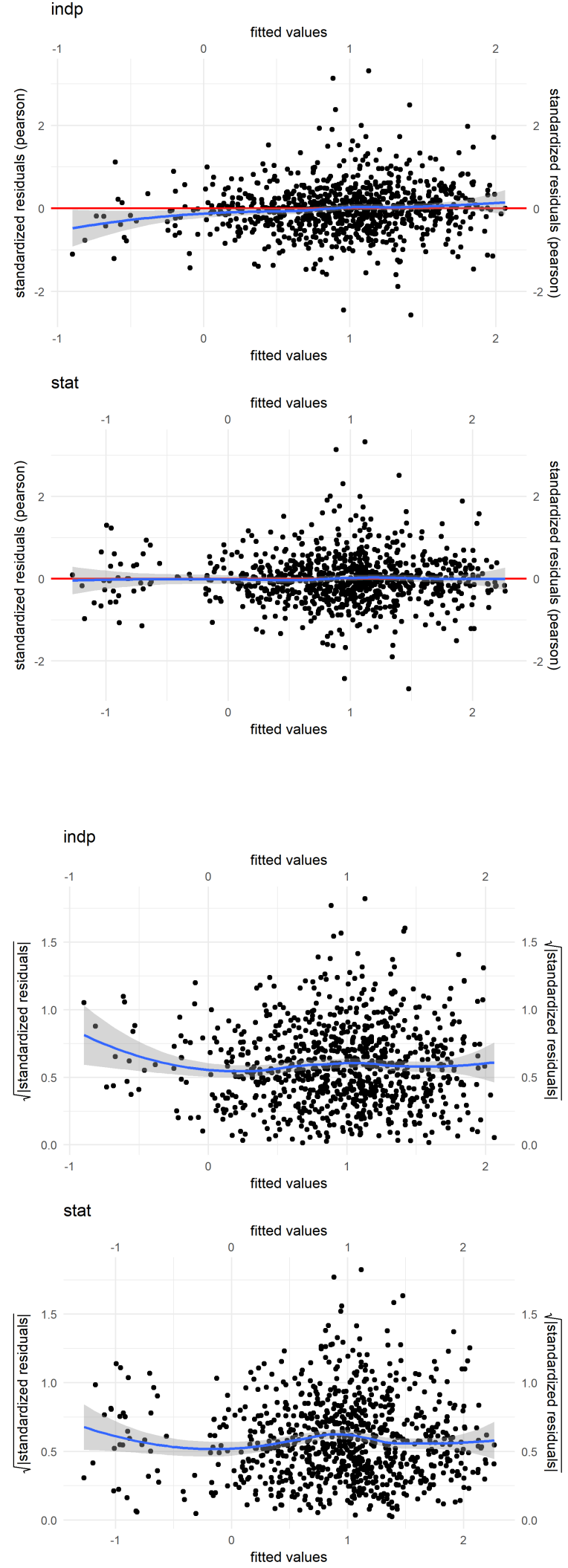
Normal-QQ (same-sex, religious service, density, state):
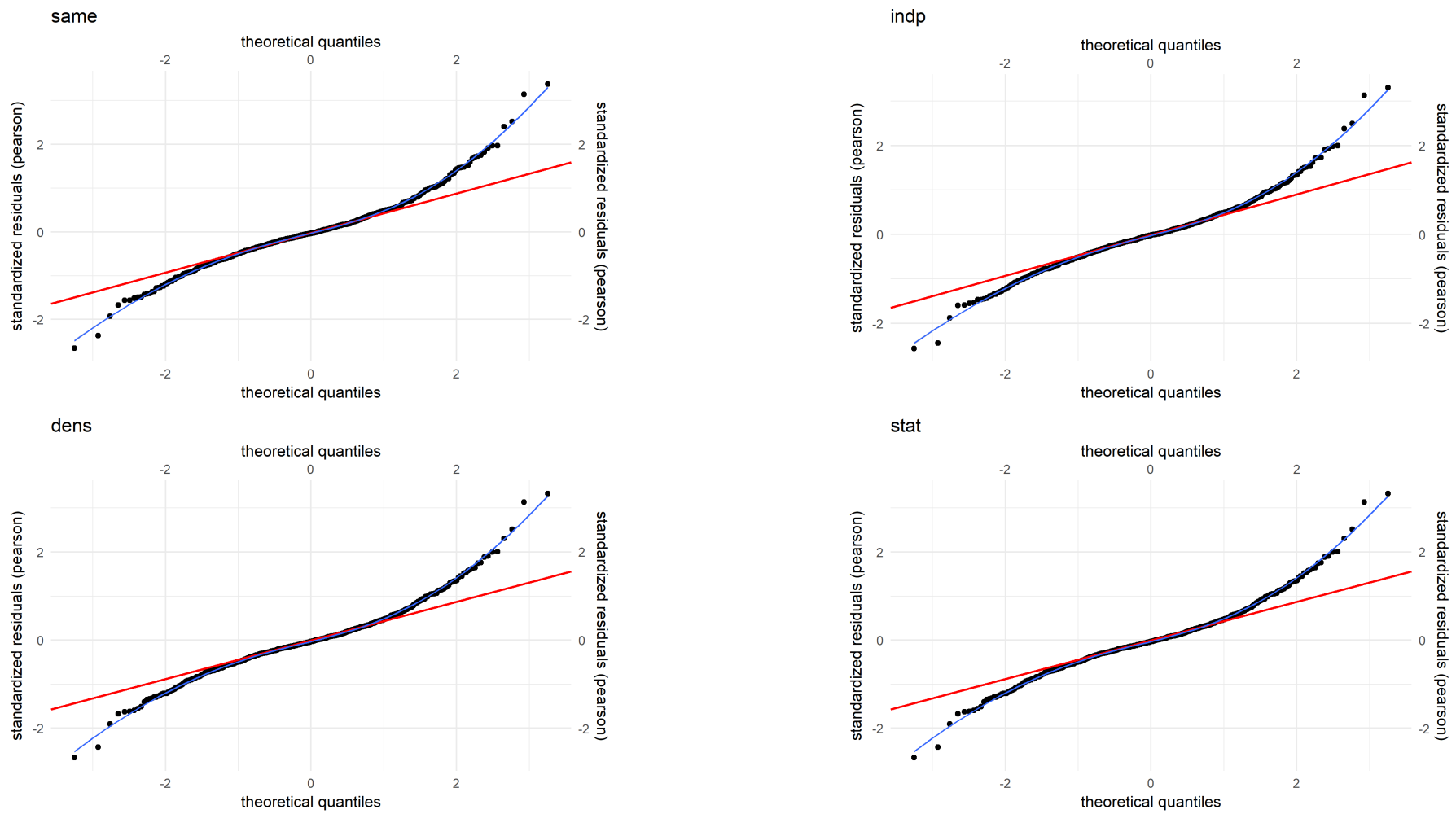

Cook's D: No influential points were excluded in the analysis. influence.ME showed that data from fully 23 observations in each model had higher Cook's D than the $4 / \mathrm{N}$ rule of thumb but none ever had Cook's D higher than the 0.5 rule of thumb. As such, we left the the data as-is. Order: same-sex, religious service, density, state:
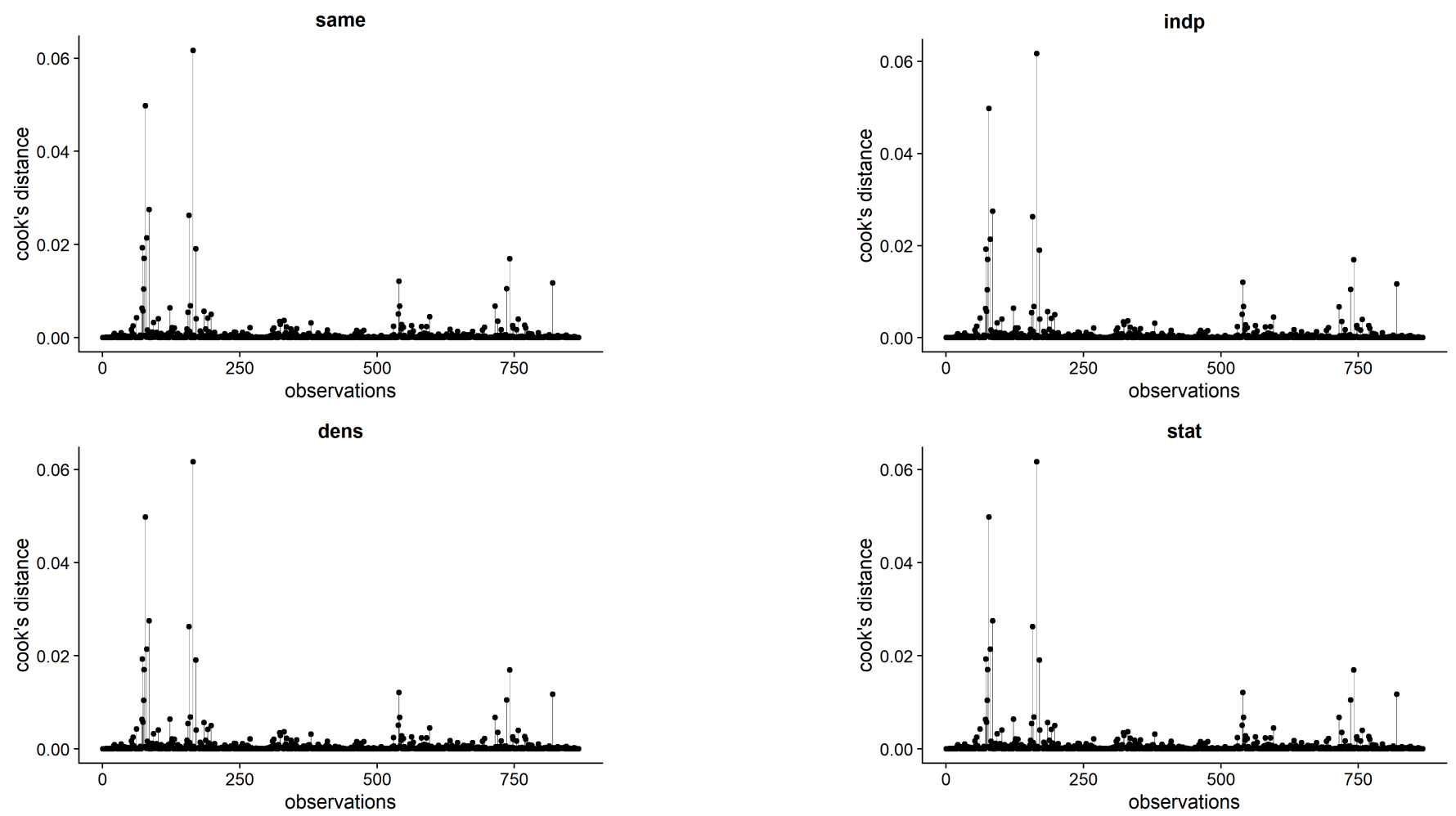